\title{
SARS-CoV-2 infection is effectively treated and prevented by EIDD-2801
}

https://doi.org/10.1038/s41586-021-03312-w

Received: 18 September 2020

Accepted: 29 January 2021

Published online: 9 February 2021

Check for updates

\author{
Angela Wahi ${ }^{1,2,3,16}$, Lisa E. Gralinski ${ }^{4,16}$, Claire E. Johnson ${ }^{1,2,3}$, Wenbo Yao ${ }^{1,2,3}$, Martina Kovarova ${ }^{1,2,3}$, \\ Kenneth H. Dinnon III ${ }^{4,5}$, Hongwei Liu ${ }^{6,7}$, Victoria J. Madden ${ }^{8}$, Halina M. Krzystek ${ }^{6,7}$, \\ Chandrav De ${ }^{1,2,3}$, Kristen K. White ${ }^{8}$, Kendra Gully ${ }^{4}$, Alexandra Schäfer ${ }^{4}$, Tanzila Zaman ${ }^{6,7}$, \\ Sarah R. Leist ${ }^{4}$, Paul O. Grant ${ }^{6,7}$, Gregory R. Bluemling ${ }^{9,10}$, Alexander A. Kolykhalov ${ }^{9,10}$, \\ Michael G. Natchus ${ }^{10}$, Frederic B. Askin ${ }^{11}$, George Painter ${ }^{9,10,12}$, Edward P. Browne ${ }^{2,3,5,13}$, \\ Corbin D. Jones ${ }^{6,7}$, Raymond J. Pickles ${ }^{5,14}$, Ralph S. Baric ${ }^{4,5,15}$ \& J. Victor Garcia ${ }^{1,2,3 凶}$
}

\begin{abstract}
All coronaviruses known to have recently emerged as human pathogens probably originated in bats ${ }^{1}$. Here we use a single experimental platform based on immunodeficient mice implanted with human lung tissue (hereafter, human lung-only mice (LoM)) to demonstrate the efficient in vivo replication of severe acute respiratory syndrome coronavirus (SARS-CoV), Middle East respiratory syndrome coronavirus (MERS-CoV) and severe acute respiratory syndrome coronavirus 2 (SARS-CoV-2), as well as two endogenous SARS-like bat coronaviruses that show potential for emergence as human pathogens. Virus replication in this model occurs in bona fide human lung tissue and does not require any type of adaptation of the virus or the host. Our results indicate that bats contain endogenous coronaviruses that are capable of direct transmission to humans. Our detailed analysis of in vivo infection with SARS-CoV-2 in human lung tissue from LoM showed a predominant infection of human lung epithelial cells, including type-2 pneumocytes that are present in alveoli and ciliated airway cells. Acute infection with SARS-CoV-2 was highly cytopathic and induced a robust and sustained type-I interferon and inflammatory cytokine and chemokine response. Finally, we evaluated a therapeutic and pre-exposure prophylaxis strategy for SARS-CoV-2 infection. Our results show that therapeutic and prophylactic administration of EIDD-2801-an oral broad-spectrum antiviral agent that is currently in phase II/III clinical trials-markedly inhibited SARS-CoV-2 replication in vivo, and thus has considerable potential for the prevention and treatment of COVID-19.
\end{abstract}

SARS-CoV-2 has recently emerged in humans and is the causative agent of COVID-19; it has resulted in substantial morbidity and mortality worldwide $^{2}$. Bats are the presumed source of SARS-CoV-2, as well as of the highly pathogenic human coronavirusesSARS-CoV and MERS-CoV ${ }^{1}$. Transmission of coronaviruses from bats to other species is well-documented and adaptation in an intermediary host can facilitate their transmission to humans ${ }^{1}$. Although it is possible that SARS-CoV-2 was transmitted to humans via an intermediate host, phylogenetic analysis has indicated that theSARS-CoV-2 lineage has circulated in bats for decades and that this virus has evolved in bats to be capable of replicating in human cells ${ }^{3}$. Given the repeated and accelerating emergence of highly pathogenic coronaviruses, it is increasingly important to monitor and characterize bat coronaviruses, and to identify the viral determinants of human infection, disease and global spread-as well as to develop effective therapeutic interventions. Animal models are useful for studying highly pathogenic human coronaviruses and the emergence potential of zoonotic coronaviruses, and for evaluating the in vivo inhibitory activity of novel agents ${ }^{4-15}$. However, human coronaviruses do not replicate in mice without extensive virus adaptation, genetic editing of the host receptor or the introduction of the appropriate human receptor genes into the host ${ }^{4,6-13,15}$. Although existing rodent models of coronavirus infection have made several important contributions, none possesses the diverse set of primary human lung cells that serve as targets for viral infection ${ }^{16}$. Here we show that immunodeficient mice implanted with authentic human lung tissue $\mathrm{e}^{17}$ (that is, LoM) allow for the in vivo study of infection withSARS-CoV, MERS-CoV or SARS-CoV-2 in a single platform that permits the direct comparison of experimental outcomes. Using

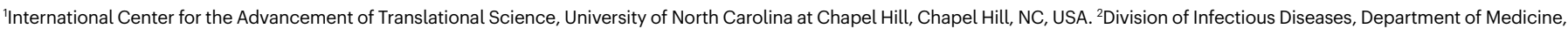
University of North Carolina at Chapel Hill, Chapel Hill, NC, USA. ${ }^{3}$ Center for AIDS Research, University of North Carolina at Chapel Hill, Chapel Hill, NC, USA. ${ }^{4}$ Department of Epidemiology,

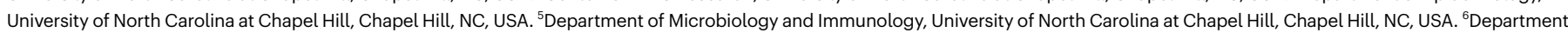
of Biology, University of North Carolina at Chapel Hill, Chapel Hill, NC, USA. ${ }^{7}$ Department of Genetics, University of North Carolina at Chapel Hill, Chapel Hill, NC, USA. ${ }^{8}$ Microscopy Services Laboratory, University of North Carolina at Chapel Hill, Chapel Hill, NC, USA. ${ }^{9}$ Emory Institute of Drug Development (EIDD), Emory University, Atlanta, GA, USA. ${ }^{10}$ Drug Innovation Ventures at Emory (DRIVE), Atlanta, GA, USA. "'Department of Pathology, University of North Carolina at Chapel Hill, Chapel Hill, NC, USA. ${ }^{12}$ Department of Pharmacology and Chemical Biology, Emory University, Atlanta, GA, USA. ${ }^{13}$ UNC HIV Cure Center, University of North Carolina at Chapel Hill, Chapel Hill, NC, USA. ${ }^{14}$ Marsico Lung Institute, University of North Carolina at Chapel, Chapel Hill, NC, USA. ${ }^{15}$ Rapidly Emerging Antiviral Drug Discovery Initiative, University of North Carolina at Chapel Hill, Chapel Hill, NC, USA. ${ }^{16}$ These authors contributed equally: Angela Wahl,

Lisa E. Gralinski. ${ }^{\bowtie}$ e-mail: victor_garcia@med.unc.edu 


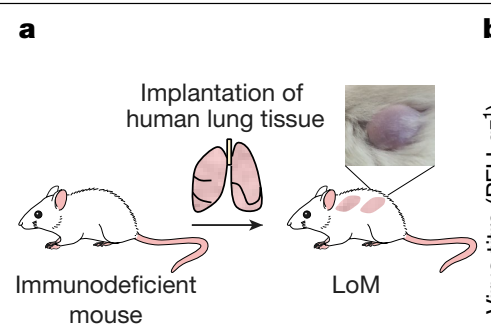

d

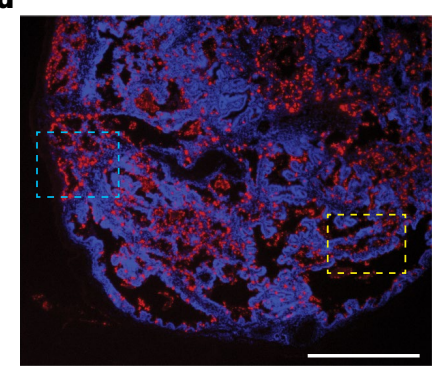

f SARS-CoV-2 NP Cytokeratin 19
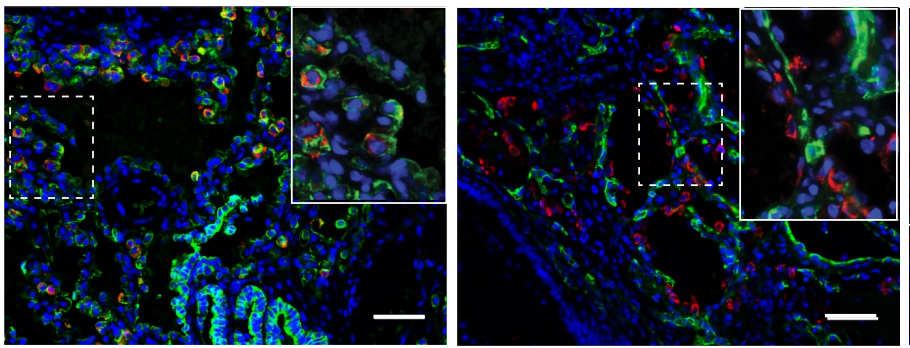

c
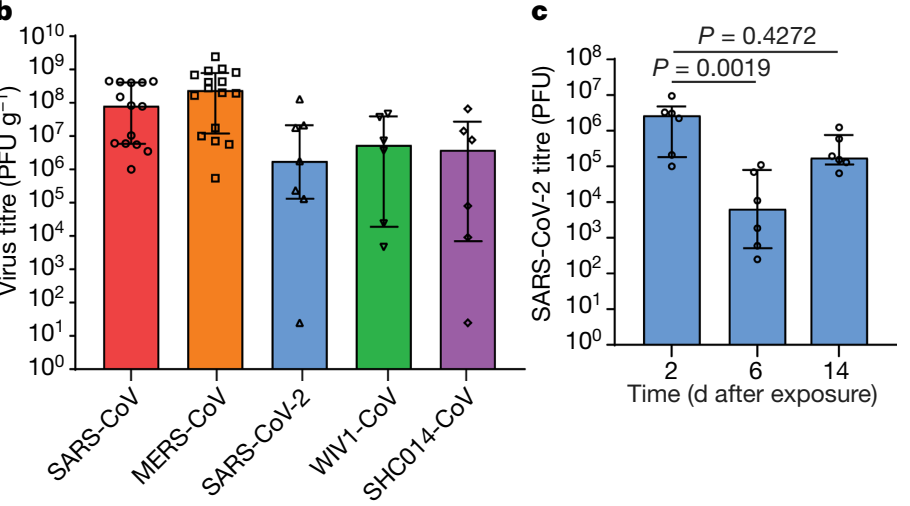
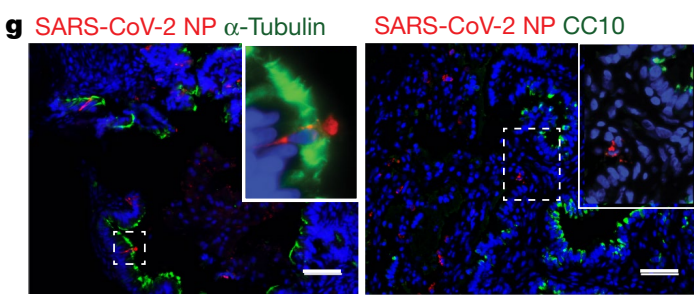

Fig. 1 | Robust replication of recently emerged human and bat coronaviruses in LoM and the predilection of SARS-CoV-2 for infection of human epithelial cells. a, LoM construction and image of a human lung implant. b, Viral titres in the human lung tissue of LoM injected with SARS-CoV $(n=14)$ (red), MERS-CoV $(n=16)$ (orange), SARS-CoV-2 $(n=7)$ (blue), WIV1-CoV $(n=6)$ (green) or SHCO14 ( $n=6)$ (purple), as determined by plaque assay. PFU, plaque-forming units. c, SARS-CoV-2 titres in the human lung tissue of LoM at days $2(n=6), 6(n=6)$ and $14(n=6)$ after exposure were compared with a two-sided Kruskal-Wallis with Dunn's multiple comparisons test.

d, SARS-CoV-2 RNA in human lung tissue of LoM at two days after exposure. SARS-CoV-2 RNA-positive, red; nuclei, blue. Scale bars, $750 \mu \mathrm{m}$ (left), $250 \mu \mathrm{m}$ (top right, bottom right). $n=3$. e, Virus nucleoprotein in human lung tissue of

LoM, we show efficient replication of bat coronaviruses in vivo without the need for virus adaptation. We also performed an in-depth in vivo analysis of acute SARS-CoV-2 infection in human lung tissue from LoM, which revealed robust virus replication, pathogenesis and sustained activation of the human innate immune response. Finally, we show that EIDD-2801-an orally administered, broad-spectrum antiviral agent that is currently in phase II/III clinical trials for the treatment of COVID-19-efficiently inhibited SARS-CoV-2 replication in human lung tissue from LoM

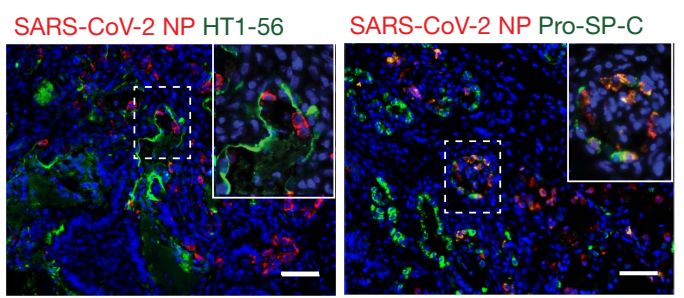

LoM two days after exposure. Nucleoprotein-positive cells, red; nuclei, blue. Scale bars, $200 \mu \mathrm{m}$ (left), $50 \mu \mathrm{m}$ (top right, bottom right). $n=6 . \mathrm{f}$, Costaining of human lung tissue of LoM, two days after exposure to SARS-CoV-2, for virus nucleoprotein (NP) (red) and cy tokeratin 19 (epithelial cells, green) $(n=6)$, CD34 (endothelial cells, green) $(n=4)$ or vimentin (mesenchymal cells, green) $(n=4)$. Nuclei, blue. Scale bars, $50 \mu \mathrm{m}$. g, Costaining of human lung tissue of LoM, two days after exposure to SARS-CoV-2, for virus nucleoprotein (red) and acetylated $\alpha$-tubulin IV (ciliated cells, green) $(n=6), \mathrm{CC10}$ (club cells, green) $(n=6), \mathrm{HT1}-56$ (alveolar type-1 pneumocytes, green) $(n=6)$ or pro-SP-C (alveolar type-2 pneumocytes, green) $(n=3)$. Nuclei, blue. Scale bars, $50 \mu \mathrm{m}$. In b, c, horizontal and vertical lines represent the median and interquartile range, respectively. $n$, number of biologically independent lung tissues analysed.

when administered therapeutically, and preventedSARS-CoV-2 infection when administered as pre-exposure prophylaxis, which together strongly support its further clinical development for COVID-19.

\section{Coronavirus replication in LoM}

LoM are constructed by the subcutaneous implantation of human lung tissue into the back of immunodeficient mice (Fig. 1a). This tissue expands 

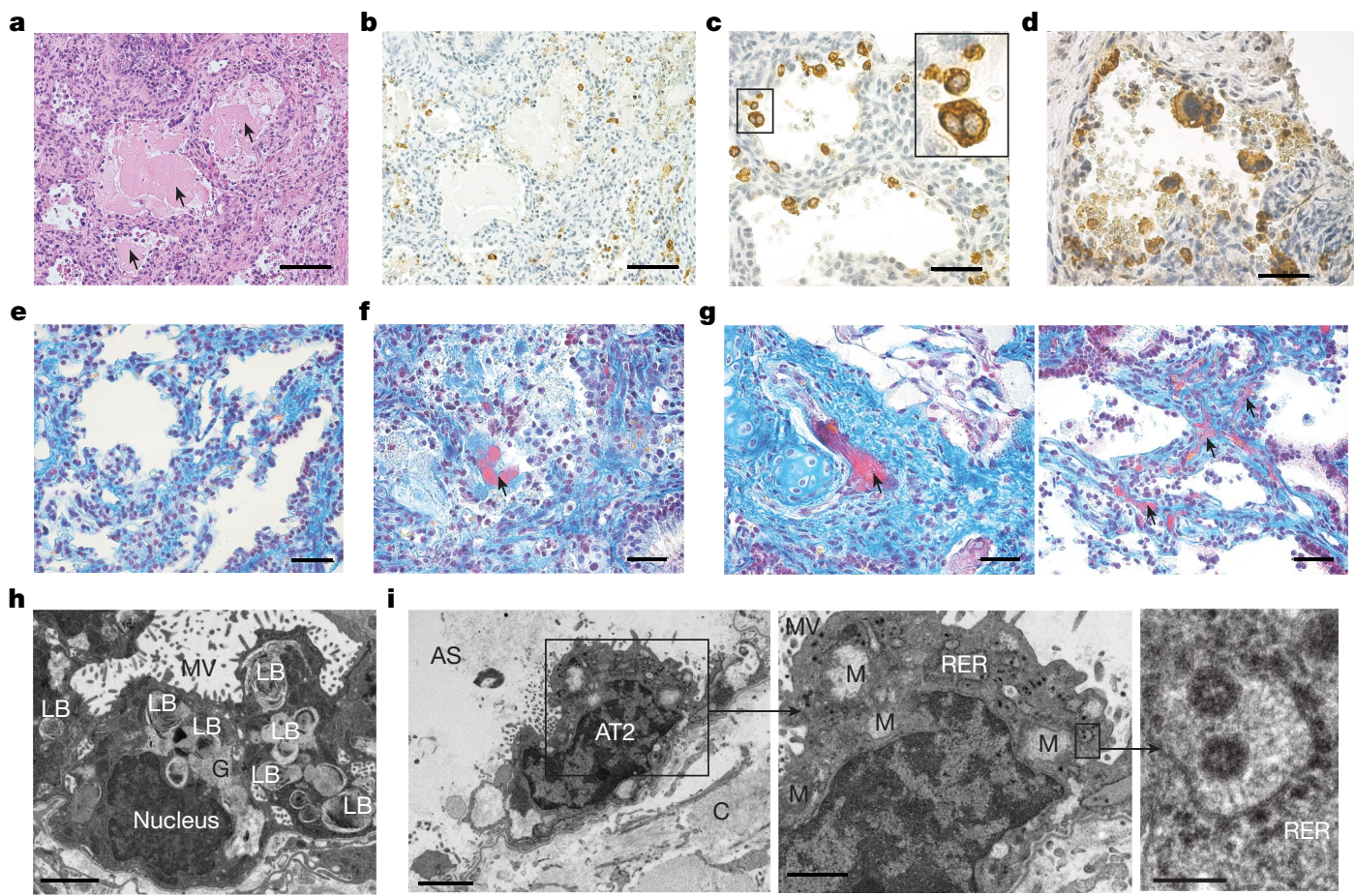
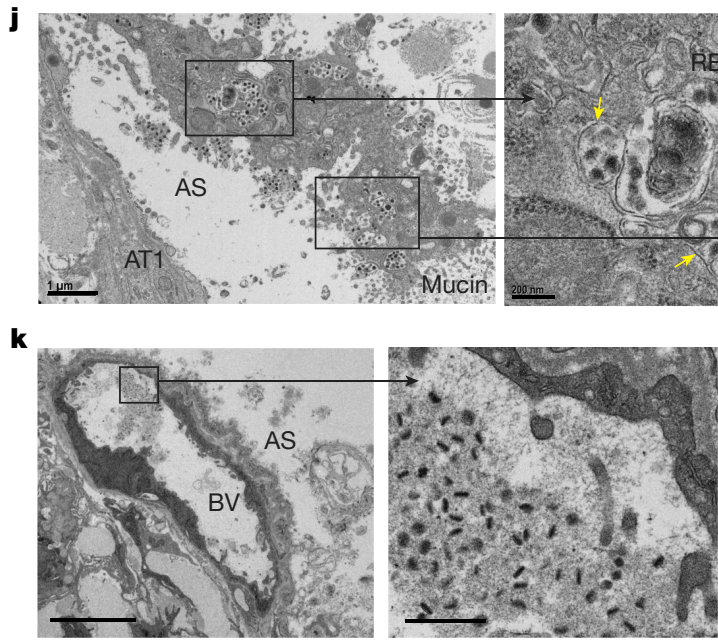

Fig. 2 | Acute SARS-CoV-2 infection is highly cytopathic and causes extensive damage to human lung structures. a, Haematoxylin and eosin (H\&E) staining of human lung tissue from a SARS-CoV-2-infected LoM two days after exposure. $n=6$. Scale bar, $100 \mu \mathrm{m}$. Arrows indicate protein globules. b-d, Immunohistochemical staining for virus nucleoprotein in human lung tissue of LoM two days after exposure to SARS-CoV-2. Nucleoprotein-positive cells, brown. Scale bars, $100 \mu \mathrm{m}(\mathbf{b}), 50 \mu \mathrm{m}(\mathbf{c}, \mathbf{d}) . n=6 . \mathbf{e}-\mathbf{g}$, Martius Scarlet Blue staining of human lung tissue from naive $\operatorname{LoM}(n=6)(\mathbf{e})$ and SARS-CoV-2 infected LoM two days after exposure $(n=6)(\mathbf{f}, \mathbf{g})$. Fibrin, red; collagen, blue. Scale bars, $50 \mu \mathrm{m}$. Arrows indicate the presence of fibrin (red) in alveoli (f) or thrombi $(\mathbf{g})$ of occluded vessels. h-l, Electron microscopy analysis of SARS-CoV-2-infected human lung tissue of LoM at two days after exposure $(n=3) . \mathbf{h}$, Uninfected alveolar type-2 pneumocytes in an alveolus-like structure.

to form a palpable implant ${ }^{17}$ (Fig. 1a). Lung implants contain human fibroblasts, epithelial, endothelial and mesenchymal cells that form cartilaginous and noncartilaginous bronchial airways that are lined with ciliated and nonciliated epithelium, alveolar sac structures and extensive vasculature $^{17}$ (Extended Data Fig. 1a,b). The human lung tissue in LoM has previously been shown to support the replication of a diverse set of emerging and clinically relevant human pathogens, including MERS-CoV ${ }^{17}$.
We evaluated the potential of LoM to serve as a single platform to study all known recently emerged human coronaviruses and the potential of endogenous bat coronaviruses for human emergence. Human angiotensin-converting enzyme 2 (ACE2), which is the receptor used by SARS-CoV and SARS-CoV-2 ${ }^{18-21}$, is expressed on human epithelial cells (cytokeratin $19^{+}$) in the human lung tissues of LoM (Extended Data Fig. 1c, d). We also confirmed the expression of transmembrane 
protease serine 2 (TMPRSS2), which primes the spike protein of SARS-CoV and SARS-CoV- $2^{18}$, in these tissues (Extended Data Fig. 1e). We inoculated LoM with SARS-CoV, MERS-CoV or SARS-CoV-2 (Extended Data Table1). Infection with SARS-CoV and SARS-CoV-2 resulted in mean virus titres of $1.76 \times 10^{8}$ and $2.42 \times 10^{7}$ plaque-forming units (PFU) $\mathrm{g}^{-1}$, respectively, at 2 days after infection (Fig. $1 b$ ). We observed abundant viral nucleoprotein antigen in the human lung tissues of LoM infected with SARS-CoV or SARS-CoV-2 (Extended Data Fig. 2a). Consistent with previous results ${ }^{17}$, MERS-CoV replicated to mean titres of $4.79 \times 10^{8} \mathrm{PFU}$ $\mathrm{g}^{-1}$ in the human lung tissues of LoM at 2 days after infection (Fig. 1b), and we observed abundant viral nucleoprotein antigen (Extended Data Fig. 2a).

The bat coronaviruses WIV1-CoV and SHC014-CoV have high sequence homology to SARS-CoV, use ACE2 to infect human cells and grow modestly in primary human airway cultures on liquid interface ${ }^{12,13}$. WIV1-CoV and SHC014-CoV efficiently replicated in the human lung tissue of LoM (Extended Data Table 1), with mean titres of $1.58 \times 10^{7}$ and $1.48 \times 10^{7} \mathrm{PFU} \mathrm{g}^{-1}$, respectively, at two days after exposure to the virus (Fig. 1b), and we readily detected viral nucleoprotein antigen in the human lung tissues (Extended Data Fig. 2b). We did not detect viral nucleoprotein antigen in the human lung tissues of naive LoM (Extended Data Fig. 2c). Collectively, these results demonstrate that LoM serve as a single platform in which the recently emerged human coronaviruses SARS-CoV, MERS-CoV and SARS-CoV-2 replicate efficiently in human lung tissue. Notably, the efficient replication of the SARS-like bat coronaviruses WIV1-CoV and SHC014-CoV that we observed is in agreement with previous in vitro data ${ }^{12,13}$, suggesting that bats contain coronaviruses that are capable of direct transmission to humans-bypassing the need for further adaptation in an intermediary host.

\section{SARS-CoV-2 replication in LoM}

We inoculated the human lung tissues of LoM with SARS-CoV-2, and determined titres of replication-competent virus at 2, 6 and 14 days after exposure (Fig. 1c, Extended Data Table 2). We noted high titres of replication-competent virus at all of the time points we measured, although titres were highest at two days after infection (Fig.1c). Infection was widely distributed throughout the tissue, and large numbers of cells were positive for viral RNA (Fig.1d) and nucleoprotein (Fig. 1e). We costained for human cytokeratin 19 , which demonstrated that SARS-CoV-2 predominantly infects human epithelial cells in the lung (Fig. 1f, Extended Data Fig. 3a). We did not detect viral nucleoprotein antigen in human CD34-expressing (endothelial) cells, and detected this antigen in only a few human vimentin-expressing (mesenchymal) cells (Fig. 1f, Extended Data Fig. 3a). We further identified the types of infected epithelial cell. We clearly identified virus nucleoprotein antigen in cells that express pro-SP-C (alveolar type-2 pneumocytes) or acetylated $\alpha$-tubulin IV (ciliated cells), but not did not detect this antigen in $\mathrm{HT} 1-56^{+}$cells (alveolar type- 1 pneumocytes) or $\mathrm{CC}^{+} 0^{+}$cells (club cells) (Fig. 1g, Extended Data Fig. 3b). These results demonstrate that SARS-CoV-2 has limited tropism in the lung, and that alveolar type-2 pneumocytes and ciliated airway epithelial cells are the predominant cells infected.

\section{SARS-CoV-2 pathogenesis in LoM}

Our histopathological analysis revealed several features of early diffuse alveolar damage that have previously been described in lung tissues of patients with COVID-19, including the accumulation of proteinaceous exudate and fibrin in alveolar spaces, desquamation of pneumocytes, multinucleated cell formation and the appearance of fibrin thrombi in small vessels ${ }^{22-24}$ (Fig. 2). We observed proteinaceous exudate-including large protein globules-in alveolar spaces that overlapped with areas of virus accumulation (Fig. 2a, b). We noted desquamation of pneumocytes as early as two days after infection; a large number of virally infected cells were fully detached or detaching from the alveolar basement membrane (Fig. 2c, d). We also observed infected multinucleated cells (Fig. 2c). Although we did not note hyaline membranes, in contrast to naive LoM (Fig. 2e), we detected fibrin in alveolar spaces in human lung tissue from SARS-CoV-2-infected LoM (Fig. 2f). Notably, we observed several occluded vessels containing fibrin thrombi, as has previously been reported in the lungs of patients with COVID-19 ${ }^{22-24}$ (Fig. 2g).Our electron microscopy investigations demonstrated that the architecture and integrity of uninfected alveolar type-2 pneumocytes in human lung tissue obtained from LoM at two days after infection were normal (Fig. 2h). By contrast, alveolar type-2 pneumocytes (from the same sample) that contained virus particles had swollen mitochondria with a loss of matrix and cristae as well as rough endoplasmic reticula with distended cisternae, protein accumulation and virus particles (Fig. 2i). We observed degenerative alveolar type-2 pneumocytes infected with SARS-CoV-2 that were detached from the alveolar basal membrane in the alveolar luminal space (Fig. 2j). A higher magnification revealed the subcellular accumulation of virus-containing vesicles, indicative of virus replication and egress. We also observed virions with electron-dense nucleocapsids and distinctive crown-like spikes (Fig. $2 \mathrm{i}$, j). Consistent with previous reports ${ }^{23,25}$, the virions produced by human lung cells were pleomorphic in size ( 69 to $112 \mathrm{~nm}$ ) and shape. Despite the extensive damage that we observed in the lung tissue, the endothelium in the majority of blood vessels was intact, and possessed tight junctions, numerous pinocytotic vesicles, and normal mitochondria and endoplasmic reticulum (Fig. $2 \mathrm{k}$, l). We did not detect virions within endothelial cells, consistent with our immunofluorescence analysis (Figs. 1f, 2k, l). However, pleomorphic virions were present in capillary lumen and were surrounded by fibrillar protein deposits and cell debris (Fig. 2k, l). Together, these results demonstrate that acute SARS-CoV-2 infection of LoM closely resembles lung infection in humans, and that it is highly cytopathic (resulting in substantial injury to the fragile alveolar lung structures).

We performed RNA-sequencing analysis of human lung tissues collected from LoM at 2, 6 and 14 days after infection. We detected abundant viral transcripts that ranged from $0.55 \%$ to $3.6 \%$ of the total reads at 2 days after infection (Extended Data Table 3); viral transcripts were abundant but lower at 6 days and 14 days after infection (Extended Data Table 3). Our sequencing data identified canonical SARS-CoV-2 transcripts ${ }^{26}$ and confirmed the maintenance of the furin cleavage site in the spike protein. Our analysis of human gene transcripts revealed 1,504 differentially expressed cellular genes between naive and infected human lung tissue from LoM at 2 days after exposure (the peak of infection) (Fig. 3a, Supplementary Tables 1,2). Our differential expression analysis suggests that 1,043 of these genes were increased in expression, and 461 were decreased, in the infected human lung tissue of LoM relative to that of naive controls (Supplementary Tables 1,2). We noted three patterns: the expression of most genes did not change with infection; many genes with increased expression in SARS-CoV-2-infected LoM were expressed at a low or moderate level in naive LoM; and a handful of genes went from undetectable expression in naive LoM to moderate or high expression in infected LoM, or from expression in naive LoM to undetectable expression in infected LoM. As expected, ACE2 and TMPRSS2 were expressed across the lung tissues (Extended Data Table 4). Notably, numerous interferon-stimulated genes and inflammatory cytokine genes-including the pro-inflammatory cytokine genes IL6, CXCL8 (which encodes IL-8), CXCL1O (which encodes IP-10), TNF and CCL5 (which encodes RANTES)-were potently induced in infected human lung tissue from LoM (Supplementary Tables 1,2). We also observed a marked upregulation of IFNB1, IFNL1, IFNL2 and IFNL3 expression ( $>1,000$-fold for all) at 2 days after exposure, which suggests that these cytokines have a key role in the antiviral response to SARS-CoV-2 (Supplementary Tables 1,2). Our gene set enrichment analysis showed that over 840 gene pathways were significantly upregulated $(P<0.05)$, 


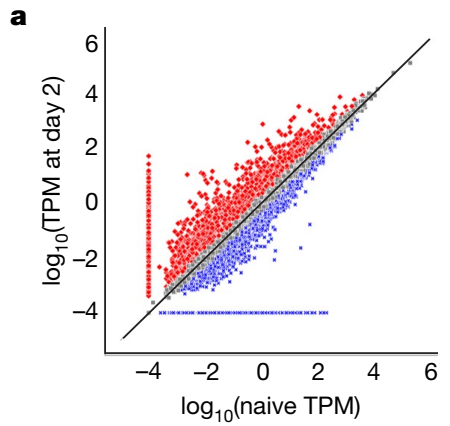

c

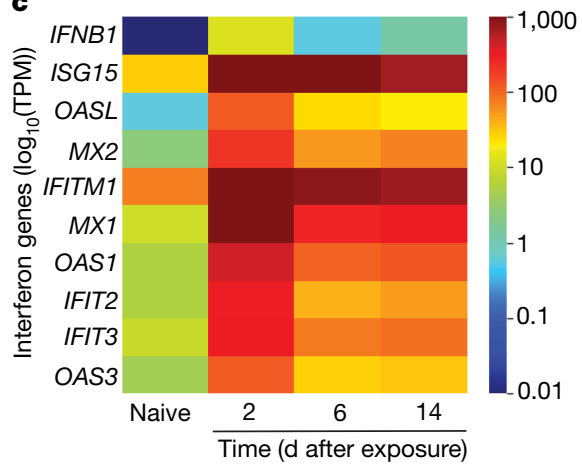

Fig. 3 |SARS-CoV-2 infection induces a strong and sustained human innate immune response in human lung tissue from LoM.a-d, RNA-sequencing analysis of human lung tissue collected from SARS-CoV-2-infected LoM. a, $\log _{10}$-transformed gene transcripts per million (TPM) in the human lungs of naive LoM $(n=2)$ ( $x$ axis) and SARS-CoV-2-infected LoM on day 2 after exposure $(n=2)$ (y axis). Genes of interest from Supplementary Tables 1, 2 that appear increased (red) or decreased (blue) by at least twofold in SARS-CoV-2-infected LoM are shown. Genes with zero (0) mean TPM in naive or infected LoM are set at a minimum of $\log _{10}(0.0001)$ TPM for visualization. b, Gene set enrichment analysis identified gene sets enriched in human lung tissue from SARS-CoV-
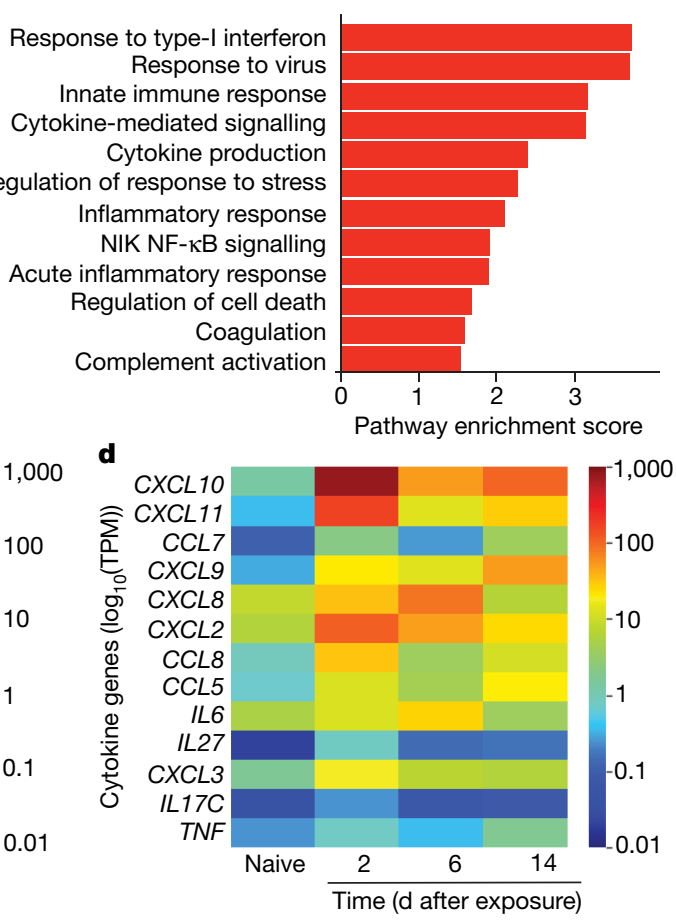

2-infected LoM. The pathway enrichment score is shown on the $x$ axis. The statistical significance of enrichment scores was determined using a two-sided empirical phenotype-based permutation test, and adjusted for multiple testing using a false discovery rate (red, $P<0.05)$. c, d, Heat maps illustrating the expression of human interferon genes (c) and cytokine and chemokine genes $(\mathbf{d})$ in human lung tissue collected from SARS-CoV-2-infected LoM on days $2(n=2), 6(n=3)$ and $14(n=3)$ after exposure, and from naive LoM $(n=4)$. Colour scale indicates the mean $\log _{10}($ TPM). $n$, number of biologically independent lung tissues analysed. including response to type-I interferon $(P=0.0011)$, response to virus $(P=0.0010)$, innate immune response $(P=0.0010)$, cytokine-mediated signalling $(P=0.0010)$, cytokine production $(P=0.0010)$, response to stress $(P=0.0010)$, inflammatory response $(P=0.0010)$, NIK NF-KB signalling $(P=0.0011)$, acute inflammatory response $(P=0.0035)$, regulation of cell death $(P=0.0030)$ and coagulation pathways $(P=0.0453)$ (Fig. 3b). Complement activation, which contributes to SARS-CoV pathogenesis in mouse models ${ }^{14}$, was also increased $(P=0.0470)$ (Fig. 3b). Importantly, our analysis of host gene expression at later time points demonstrated a sustained upregulation of antiviral and inflammatory genes that-in some instances (for example, ISG15, IFITM1, $T N F$ and $C X C L 9$ ) - persisted for up to 14 days after infection (the final time point that we analysed) (Fig. 3c, d, Extended Data Table 5, Supplementary Tables 1,2). These results demonstrate that acute SARS-CoV-2 infection causes a potent and sustained upregulation of innate immune responses in virus-infected human lung tissue of LoM.

\section{EIDD-2801 treatment and prophylaxis}

The ribonucleoside analogue $\beta$-D- $N^{4}$-hydroxycytidine (NHC) broadly inhibits coronavirus infection in vitro in cultures of human airway epithelial cells ${ }^{15}$. Prophylactic and therapeutic administration of the oral pro-drug of NHC (EIDD-2801; also known as molnupiravir or MK-4482) reduced SARS-CoV and MERS-CoV replication and pathogenesis in mice ${ }^{15} .5^{\prime}$-triphosphate $\mathrm{NHC}$ acts as a competitive alternate substrate for the viral RNA-dependent RNA polymerase, which allows the incorporation of 5 '-triphosphate NHC into viral RNA and results in the accumulation of mutations within the viral RNA genome that lead to error catastrophe $\mathrm{e}^{15}$. As an orally bioavailable agent, EIDD-2801 can be administered to patients much more readily than can remdesivir and other antiviral and biological agents (for example, convalescent plasma and monoclonal antibodies), which require administration by infusion in a clinical setting. We tested the ability of therapeutic use of EIDD-2801 to inhibit SARS-CoV-2 replication in vivo in LoM, using a dose that is similar to the human dose in clinical trials ${ }^{27}$. We administered EIDD-2801 to LoM starting at 24 or $48 \mathrm{~h}$ after exposure to SARS-CoV-2, and every $12 \mathrm{~h}$ thereafter (Extended Data Fig. 4a, Extended Data Table 6). Our results show that EIDD-2801 had a notable effect on virus replication after only two days of treatment (Fig. 4a, b). EIDD-2801 markedly reduced the number of infectious particles in the human lung tissue of LoM by 4.4 logs (>25,000-fold decrease) when treatment was initiated at $24 \mathrm{~h}$ after exposure $(P=0.0002)$ (Fig. 4a). When treatment was started at $48 \mathrm{~h}$ after exposure, virus titres were significantly reduced by $96 \%$ (1.5 logs, $P=0.0019)$ (Fig. $4 \mathrm{~b})$. Next, we tested the efficacy of pre-exposure prophylaxis using EIDD-2801. We administered EIDD2801 to LoM starting $12 \mathrm{~h}$ before exposure to SARS-CoV-2, and every $12 \mathrm{~h}$ thereafter (Extended Data Fig. 4b, Extended Data Table 6). EIDD2801 pre-exposure prophylaxis significantly reduced virus titres in the human lung tissues of LoM by over 100,000-fold in 2 independent experiments $(P=0.0002$ and $P=0.0068)$ (Fig. $4 \mathrm{c}-\mathrm{e})$. Furthermore, in contrast to the situation in mice treated with EIDD-2801, we observed abundant cell debris and nucleoprotein-positive cells in the alveolar lumen of mice treated with vehicle control, consistent with the extensive pathogenic effects inflicted on the lung by SARS-CoV-2 (Fig. 4f, g). 

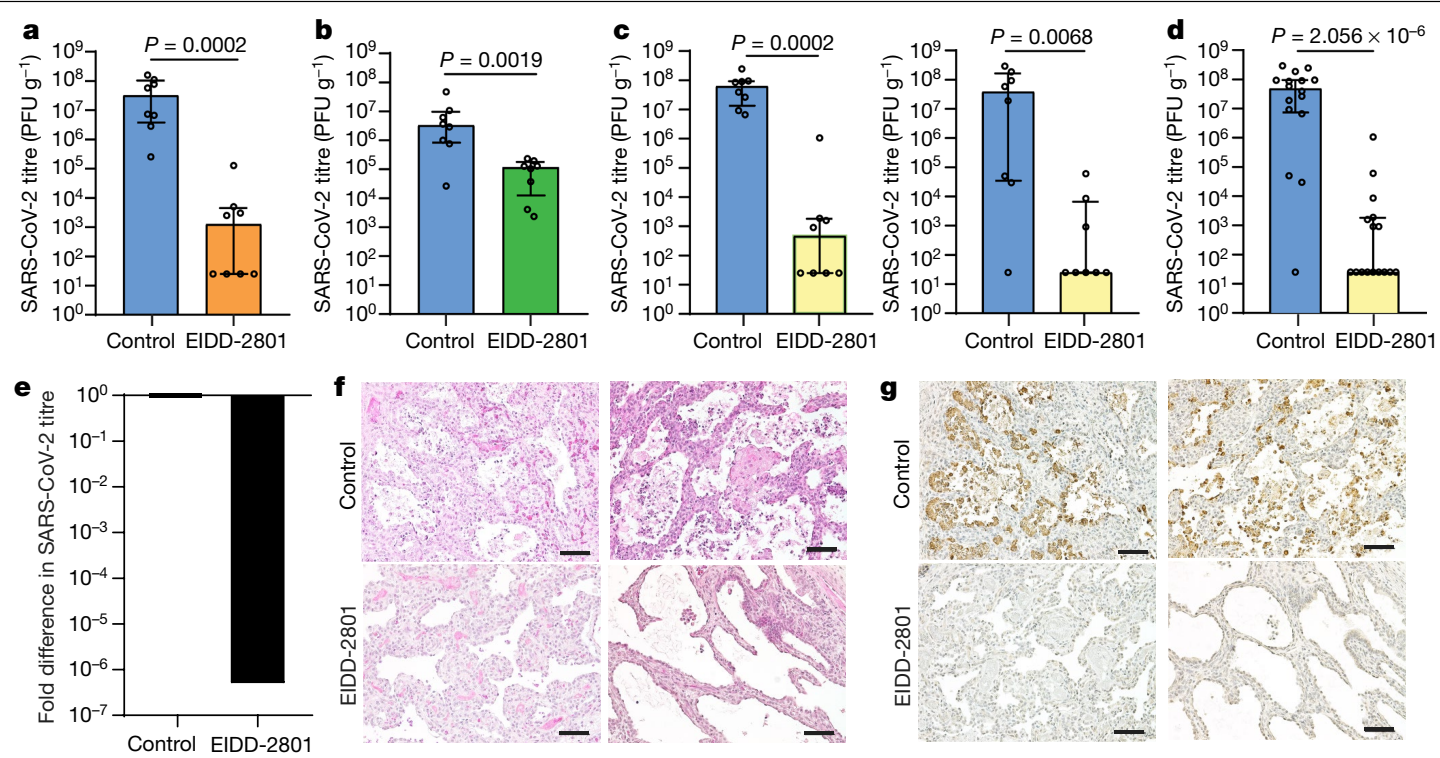

Fig. 4 | Treatment and pre-exposure prophylaxis with EIDD-2801 potently inhibit SARS-CoV-2 infection in vivo. a, SARS-CoV-2 titres in the human lung tissue of LoM administered EIDD-2801 $(n=8)$ or vehicle control $(n=8) 24 \mathrm{~h}$ after exposure to virus. $\mathbf{b}$, SARS-CoV-2 titres in the human lung tissue of LoM administered EIDD-2801 $(n=8)$ or vehicle $(n=8) 48 \mathrm{~h}$ after exposure to virus. c, d, SARS-CoV-2 titres in the human lung tissue of LoM administered EIDD-2801 ( $n=8$ per experiment) or control vehicle ( $n=8$ per experiment) $12 \mathrm{~h}$ before exposure to virus in two independent experiments shown separately (c) and combined (d).e, Fold difference in SARS-CoV-2 titres in the human lung

These results demonstrate that prophylactic administration of EIDD-2801 is highly effective at preventing SARS-CoV-2 infection and pathogenesis in vivo in the human lung tissues of LoM.

\section{Discussion}

Our results demonstrate replication of all known recently emerged human coronaviruses in LoM. Importantly, consistent with in vitro studies $^{12,13}$, our results demonstrate the relatively efficient replication of two endogenous bat viruses in the human lung tissue of LoM in vivo, which indicates that coronaviruses circulating in bats have pandemic potential without the need for further adaptation to humans. Acute SARS-CoV-2 infection of LoM resulted in substantial lung injury and exhibited key features of the extensive lung pathology observed in patients with severe COVID-19 ${ }^{22,24,28,29}$. Consistent with analyses of bronchoalveolar fluid or post-mortem lung samples obtained from patients with COVID-19 $9^{5,30}$, several interferon-stimulated genes were significantly increased in SARS-CoV-2-infected human lung tissue from LoM. We observed a robust induction of IFNB1 expression during acute infection, followed by a decline in expression. A post-mortem analysis of the lungs of patients with COVID- $19^{5} \mathrm{did}$ not reveal increased IFNB1 expression, and it has previously been shown in vitro that the expression of IFNB1 is blocked during SARS-CoV infection $^{31,32}$. These results suggest that, in human lung tissue, IFNB1 expression is induced during acute SARS-CoV-2 infection. Although this study was not designed to evaluate the effect of type-I interferon on SARS-CoV-2 replication in vivo, in vitro studies and in vivo mouse studies have shown that treatment with type-I interferon restricts SARS-CoV-2 replication ${ }^{7,33}$. We also observed an increased expression of several human cytokine genes in SARS-CoV-2-infected LoM, many of which have also been shown to be increased in analyses of serum and post-mortem lung tissue from patients with COVID-19-further establishing the similarities between LoM and human SARS-CoV-2 infection ${ }^{5,34}$. tissue of LoM administered EIDD-280112 $\mathrm{h}$ before exposure to virus relative to vehicle controls.f, $\mathbf{g}, \mathrm{H} \&$ E staining (f) and immunohistochemical staining (g) for virus nucleoprotein (nucleoprotein-positive cells, brown) of human lung tissue of LoM administered EIDD-2801 $(n=8)$ or control vehicle $(n=8) 12 \mathrm{~h}$ before exposure to virus. Scale bars, $100 \mu \mathrm{m}$. a-d, Titres were compared with a two-tailed Mann-Whitney $U$ test. Horizontal and vertical lines represent the median and interquartile range, respectively. $n$, number of biologically independent lung tissues analysed.

The SARS-CoV-2 vaccines that have been authorized for emergency use by the US Food and Drug Administration are highly effective at preventing disease. However, it may take considerable time to reach the target vaccination levels that are needed for herd immunity (especially in resource-limited settings), owing to manufacturing capabilities, required vaccine shipping and storage conditions, and public acceptance. Therefore, alternative treatments and preventive approaches are still urgently needed. Remdesivir is limited to use in hospitalized patients, and its effectiveness at reducing disease and mortality late in infection is not clear ${ }^{35,36}$. Although a carefully controlled clinical trial sponsored by the National Institutes of Health has reported that remdesivir significantly shortened the time of recovery ${ }^{35}$, the SOLIDARITY trial of the World Health Organization revealed no significant reduction in the duration of hospitalization or mortality ${ }^{36}$. The early administration of the monoclonal antibody therapies bamlanivimab and REGN-CoV2 significantly reduced viral loads in patients with COVID-19 who exhibited mild-to-moderate disease ${ }^{37,38}$. However, these agents must be administered by infusion in a clinical setting, which limits their potential for widespread use. A highly efficacious antiviral agent that is administered orally has the potential for more widespread use, and for administration to patients with mild-to-moderate disease $^{39}$. Here we show that EIDD-2801 administered therapeutically to LoM for only two days significantly reduced infectious virus titres in vivo by over 4 logs; the sooner that the EIDD-2801 treatment was initiated after exposure to SARS-CoV-2 exposure, the greater was the reduction in virus replication. We also observed that prophylactic administration of EIDD-2801 efficiently prevents SARS-CoV-2 infection in vivo, which highlights the potential utility of EIDD-2801 as an effective prophylactic and therapeutic agent against SARS-CoV-2 (as well as, potentially, other past and future zoonotic coronaviruses). NHC belongs to a class of ribonucleoside analogues that are known to often affect mitochondrial replication and function. However, NHC did not cause significant mitochondrial toxicity or impair mitochondrial function in vitro ${ }^{40}$. Furthermore, no significant increase 
in transition frequencies in nuclear or mitochondrial message was detected in the lung tissue of ferrets dosed with EIDD-2801 for seven days, consistent with the observed good tolerability of the drug ${ }^{41}$. In ferrets, therapeutic administration of EIDD-2801 led to a reduction in SARS-CoV-2 titres in nasal passages and prevented transmission to co-housed naive animals ${ }^{42}$. Phase II and phase II/III clinical trials are ongoing to evaluate the safety of EIDD-2801 and its effect on viral shedding using doses up to $1,600 \mathrm{mg}$ per day, a dose that is expected to provide similar intracellular levels of $5^{\prime}$-triphosphate NHC to those of a dose of 300-500 mg per kg body weight in mice. As with any antiviral agent, the therapeutic and prophylactic use of EIDD-2801 will be dictated by risk-benefit analyses. The limitations of our study include the absence of human nasal airway structures in LoM; these are thought to be early sites of SARS-CoV-2 replication in humans ${ }^{43}$. Because LoM do not have an autologous human adaptive immune system, they reflect the direct effect of viruses on their targets and bystander cells as well as the innate immune response to infection. Collectively, our results demonstrate the utility of LoM as a single in vivo platform to evaluate and compare the replication and pathogenesis of past, present and future pre-emergent, epidemic and pandemic coronaviruses, which will allow for accelerating the development and testing of therapeutic and pre-exposure prophylaxis agents such as EIDD-2801.

\section{Online content}

Any methods, additional references, Nature Research reporting summaries, source data, extended data, supplementary information, acknowledgements, peer review information; details of author contributions and competing interests; and statements of data and code availability are available at https://doi.org/10.1038/s41586-021-03312-w.

1. Cui, J., Li, F. \& Shi, Z. L. Origin and evolution of pathogenic coronaviruses. Nat. Rev. Microbiol. 17, 181-192 (2019)

2. Dong, E., Du, H. \& Gardner, L. An interactive web-based dashboard to track COVID-19 in real time. Lancet Infect. Dis. 20, 533-534 (2020).

3. Boni, M. F. et al. Evolutionary origins of the SARS-CoV-2 sarbecovirus lineage responsible for the COVID-19 pandemic. Nat. Microbiol. 5, 1408-1417 (2020).

4. Bao, L. et al. The pathogenicity of SARS-CoV-2 in hACE2 transgenic mice. Nature 583, 830-833 (2020)

5. Blanco-Melo, D. et al. Imbalanced host response to SARS-CoV-2 drives development of COVID-19. Cell 181, 1036-1045 (2020).

6. Cockrell, A. S. et al. A mouse model for MERS coronavirus-induced acute respiratory distress syndrome. Nat. Microbiol. 2, 16226 (2017).

7. Dinnon, K. H. III et al. A mouse-adapted model of SARS-CoV-2 to test COVID-19 countermeasures. Nature 586, 560-566 (2020).

8. Gralinski, L. E. et al. Complement activation contributes to severe acute respiratory syndrome coronavirus pathogenesis. MBio 9, e01753-18 (2018).

9. Jiang, R. D. et al. Pathogenesis of SARS-CoV-2 in transgenic mice expressing human angiotensin-converting enzyme 2. Cell 182, 50-58.e8 (2020).

10. McCray, P. B. Jr et al. Lethal infection of K18-hACE2 mice infected with severe acute respiratory syndrome coronavirus. J. Virol. 81, 813-821 (2007).

11. Menachery, V. D. et al. Middle East respiratory syndrome coronavirus nonstructural protein 16 is necessary for interferon resistance and viral pathogenesis. MSphere 2, e00346-17 (2017).

12. Menachery, V. D. et al. A SARS-like cluster of circulating bat coronaviruses shows potential for human emergence. Nat. Med. 21, 1508-1513 (2015).

13. Menachery, V. D. et al. SARS-like WIV1-CoV poised for human emergence. Proc. Natl Acad. Sci. USA 113, 3048-3053 (2016).

14. Rockx, B. et al. Comparative pathogenesis of COVID-19, MERS, and SARS in a nonhuman primate model. Science 368, 1012-1015 (2020).
15. Sheahan, T. P. et al. An orally bioavailable broad-spectrum antiviral inhibits SARS-CoV-2 in human airway epithelial cell cultures and multiple coronaviruses in mice. Sci. Transl. Med. 12, eabb5883 (2020).

16. Franks, T. J. et al. Resident cellular components of the human lung: current knowledge and goals for research on cell phenotyping and function. Proc. Am. Thorac. Soc. 5, 763-766 (2008)

17. Wahl, A. et al. Precision mouse models with expanded tropism for human pathogens. Nat. Biotechnol. 37, 1163-1173 (2019).

18. Hoffmann, M. et al. SARS-CoV-2 cell entry depends on ACE2 and TMPRSS2 and is blocked by a clinically proven protease inhibitor. Cell 181, 271-280 (2020).

19. Li, W. et al. Angiotensin-converting enzyme 2 is a functional receptor for the SARS coronavirus. Nature 426, 450-454 (2003)

20. Walls, A. C. et al. Structure, function, and antigenicity of the SARS-CoV-2 spike glycoprotein. Cell 181, 281-292 (2020).

21. Yan, R. et al. Structural basis for the recognition of SARS-CoV-2 by full-length human ACE2. Science 367, 1444-1448 (2020).

22. Carsana, L. et al. Pulmonary post-mortem findings in a series of COVID-19 cases from northern Italy: a two-centre descriptive study. Lancet Infect. Dis. 20, 1135-1140 (2020).

23. Menter, T. et al. Postmortem examination of COVID-19 patients reveals diffuse alveolar damage with severe capillary congestion and variegated findings in lungs and other organs suggesting vascular dysfunction. Histopathology 77, 198-209 (2020).

24. Tian, S. et al. Pulmonary pathology of early-phase 2019 novel coronavirus (COVID-19) pneumonia in two patients with lung cancer. J. Thorac. Oncol. 15, 700-704 (2020).

25. Zhu, N. et al. A novel coronavirus from patients with pneumonia in China, 2019. N. Engl. J. Med. 382, 727-733 (2020).

26. Kim, D. et al. The architecture of SARS-CoV-2 transcriptome. Cell 181, 914-921 (2020).

27. Merck Sharp \& Dohme Corp. Efficacy and Safety of Molnupiravir (MK-4482) in Hospitalized Adult Participants With COVID-19 (MK-4482-001), https://ClinicalTrials.gov/show/ NCT04575584 (2020).

28. Xu, Z. et al. Pathological findings of COVID-19 associated with acute respiratory distress syndrome. Lancet Respir. Med. 8, 420-422 (2020).

29. Zhang, $\mathrm{H}$. et al. Histopathologic changes and SARS-CoV-2 immunostaining in the lung of a patient with COVID-19. Ann. Intern. Med. 172, 629-632 (2020).

30. Zhou, Z. et al. Heightened innate immune responses in the respiratory tract of COVID-19 patients. Cell Host Microbe 27, 883-890 (2020).

31. Siu, K. L. et al. Severe acute respiratory syndrome coronavirus M protein inhibits type I interferon production by impeding the formation of TRAF3.TANK.TBK1/IKKE complex. J. Biol. Chem. 284, 16202-16209 (2009).

32. Spiegel, M. et al. Inhibition of beta interferon induction by severe acute respiratory syndrome coronavirus suggests a two-step model for activation of interferon regulatory factor 3. J. Virol. 79, 2079-2086 (2005).

33. Vanderheiden, A et al. Type I and type III interferons restrict SARS-CoV-2 infection of human airway epithelial cultures. J. Virol. 94, e00985-20 (2020).

34. Qin, C. et al. Dysregulation of immune response in patients with coronavirus 2019 (COVID-19) in Wuhan, China. Clin. Infect. Dis. 71, 762-768 (2020)

35. Beigel, J. H. et al. Remdesivir for the treatment of Covid-19 - final report. N. Engl. J. Med. 383, 1813-1826 (2020)

36. WHO Solidarity Trial Consortium. Repurposed antiviral drugs for COVID-19 -interim WHO Solidarity trial results. N. Engl. J. Med. 384, 497-511 (2021).

37. Chen, P. et al. SARS-CoV-2 neutralizing antibody LY-CoV555 in outpatients with Covid-19. N. Engl. J. Med. 384, 229-237 (2021).

38. Regeneron Pharmaceuticals Inc. Regeneron's COVID-19 outpatient trial prospectively demonstrates that REGN-COV2 antibody cocktail significantly reduced virus levels and need for further medical attention. https://newsroom.regeneron.com/news-releases/ news-release-details/regenerons-covid-19-outpatient-trial-prospectively-demonstrates (2020).

39. Kim, P. S., Read, S. W. \& Fauci, A. S. Therapy for early COVID-19: a critical need. J. Am. Med. Assoc. 324, 2149-2150 (2020).

40. Sticher, Z. M. et al. Analysis of the potential for $N^{4}$-hydroxycytidine to inhibit mitochondrial replication and function. Antimicrob. Agents Chemother. 64, e01719-19 (2020).

41. Toots, M. et al. Characterization of orally efficacious influenza drug with high resistance barrier in ferrets and human airway epithelia. Sci. Transl. Med. 11, eaax5866 (2019).

42. Cox, R. M., Wolf, J. D. \& Plemper, R. K. Therapeutically administered ribonucleoside analogue MK-4482/EIDD-2801 blocks SARS-Cov-2 transmission in ferrets. Nat. Microbiol. 6, 11-18 (2021).

43. Hou, Y. J. et al. SARS-CoV-2 reverse genetics reveals a variable infection gradient in the respiratory tract. Cell 182, 429-446 (2020).

Publisher's note Springer Nature remains neutral with regard to jurisdictional claims in published maps and institutional affiliations.

(c) The Author(s), under exclusive licence to Springer Nature Limited 2021 


\section{Methods}

No statistical methods were used to predetermine sample size. The experiments were not randomized, and investigators were not blinded to allocation during experiments and outcome assessment.

\section{Ethics statement}

Mouse studies were carried out according to protocols approved by the Institutional Use and Care Committee at UNC Chapel Hill and in adherence to the NIH Guide for the Care and Use of Laboratory Animals. Mice were kept on a 12-h light/12-h dark cycle and housed in a temperature $\left(20-23^{\circ} \mathrm{C}\right)$ - and humidity (30-70\%)-controlled vivarium maintained by the Division of Comparative Medicine at the University of North Carolina Chapel Hill.

\section{Experimental design}

LoM were used as an in vivo model to evaluate the infection of lung tissue with the recombinant coronaviruses SARS-CoV, MERS-CoV and SARS-CoV-2, as well as the full-length bat coronaviruses WIV1-CoV and SHC014-CoV ${ }^{12,13,44,45}$. Viruses were directly injected into the human lung tissue of LoM, and lung tissue was collected 2, 6 or 14 days after exposure to determine virus titre and/or for analysis by histology, electron microscopy or RNA sequencing.

\section{Construction of humanized mice}

LoM were constructed with 1 or 2 human lung implants by surgically implanting human lung tissue (Advanced Bioscience Resources) subcutaneously into the upper and lower back of male and female

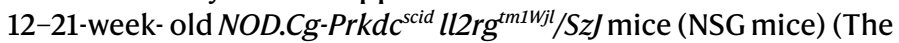
Jackson Laboratory), as previously described ${ }^{17}$. Engraftment of lung tissue was assessed by palpation, and by eight weeks after surgery mice were ready for experimentation.

Production of coronavirus stocks and infection of humanized mice Stocks of wild-type SARS-CoV, MERS-CoV (HCoV-EMC/2012), SHC014-CoV and WIV1-CoV were derived from infectious virus clones and were prepared and titred on Vero E6 (SARS-CoV, SHC014-CoV and WIV1-CoV) or Vero CCL81 cells (MERS-CoV) (American Type Culture Collection), as previously described ${ }^{6,12,13,17}$. Cell lines were authenticated by morphological identification and virus susceptibility profiles, and tested for mycoplasma by the supplier. A clinical isolate of SARS-CoV-2 (2019-nCoV/USA-WA1/2020) was obtained from the US Centers for Disease Control and Prevention (GenBank accession number MN985325.1) and passaged twice in Vero E6 cells to create a passage- 5 working stock ${ }^{43}$. For the infection of mice with coronavirus, the fur over the human lung tissue of the anaesthetized LoM was shaved and virus $\left(1-3 \times 10^{5} \mathrm{PFU}\right.$ in $100 \mu \mathrm{l}$ PBS) was injected directly into the lung tissue. To evaluate the in vivo inhibitory activity of pre-exposure prophylaxis using EIDD-2801, mice were administered $500 \mathrm{mg} \mathrm{kg}^{-1}$ EIDD-2801 or vehicle control (10\% PEG and 2.5\% Cremophor RH40 in water) via oral gavage starting $12 \mathrm{~h}$ before exposure to SARS-CoV-2, and every $12 \mathrm{~h}$ thereafter. The therapeutic efficacy of EIDD-2801 was assessed by administering mice 500 $\mathrm{mg} \mathrm{kg}^{-1}$ EIDD-2801 or vehicle control (10\% PEG and 2.5\% Cremophor $\mathrm{RH} 40$ in water) via oral gavage starting $24 \mathrm{~h}$ or $48 \mathrm{~h}$ after exposure, and every $12 \mathrm{~h}$ thereafter. At necropsy, human lung tissues of LoM were collected, weighed (mean weight of $0.55 \pm 0.05 \mathrm{~g}( \pm$ s.e.m.); $n=32$ ), homogenized, and stored at $-80^{\circ} \mathrm{C}$ until titring on Vero E6 cells. Titres below the limit of the assay $\left(50 \mathrm{PFU} \mathrm{m}{ }^{-1}\right)$ were assigned a value of 25 $\mathrm{PFU} \mathrm{g}^{-1}$. No correlation was observed between SARS-CoV-2 titre (PFU $\left.\mathrm{g}^{-1}\right)$ at 2 days after exposure and the timing after surgery $(P=0.4474$, two-tailed Spearman's rank correlation).

\section{H\&E staining of human lung tissue from LoM}

Human lung tissues collected from LoM were fixed in $10 \%$ formalin, paraffin-embedded and cut into $5-\mu \mathrm{m}$ sections, which were mounted onto Superfrost Plus slides (Fisher Scientific). Tissue sections were incubated at $60^{\circ} \mathrm{C}$ for $1 \mathrm{~h}$, deparaffinized with xylene $(2 \times 3 \mathrm{~min})$ and graded ethanol (100\% $2 \times 3 \mathrm{~min}, 95 \% 1 \times 3 \mathrm{~min}, 80 \% 1 \times 3 \mathrm{~min}, 70 \% 1 \times 3$ min), and stained with haematoxylin followed by eosin. Tissue sections were then mounted and imaged on a Nikon Eclipse Ci microscope using Nikon Elements BR software (version 4.30.01) with a Nikon Digital Sight DS-Fi2 camera. Brightness, contrast and white balance were adjusted on whole images in Adobe Photoshop (CS6).

\section{Immunohistochemical analysis of coronavirus infection}

Immunohistochemistry was performed as previously described ${ }^{17}$. In brief, fixed (10\% formalin) human lung tissues collected from coronavirus-infected LoM were paraffin-embedded and sectioned $(5 \mu \mathrm{m})$. Tissue sections mounted on Superfrost Plus slides (Fisher Scientific) were deparaffinized as described in 'H\&E staining of human lung tissue from LoM'. Following antigen retrieval using Diva Decloaker (BioCare Medical), nonspecific binding was blocked using Background Sniper (BioCare Medical). Tissue sections were then incubated with primary antibodies against SARS-CoV nucleocapsid (1:500 or 1:1,000), MERS-CoV nucleocapsid (1:2,000) or TMPRSS2 (1:100) overnight at $4{ }^{\circ} \mathrm{C}$. Tissue sections incubated with rabbit IgG were used as isotype controls. Tissue sections were then washed in TBST and the endogenous peroxidase activity was blocked with hydrogen peroxide. Tissue sections were developed using the $\mathrm{MACH}-3$ polymer system (BioCare Medical) and 3,3'-diaminobenzidine (DAB) (Vector Laboratories), counterstained with haematoxylin and mounted. Tissue sections were imaged on a Nikon Eclipse Ci microscope using Nikon Elements BR software (version 4.30.01) with a Nikon Digital Sight DS-Fi2 camera. Adobe Photoshop (CS6) was used to adjust brightness, contrast and white balance on whole images.

\section{Immunofluorescence analysis of SARS-CoV-2 infection}

Human lung tissues collected from LoM were fixed in $10 \%$ formalin and paraffin-embedded. Immunofluoresence staining of 5- $\mu \mathrm{m}$ tissue sections was performed as previously described ${ }^{17}$. In brief, following deparaffinization and antigen retrieval (Diva Decloaker), tissue sections were incubated with a $10 \%$ normal donkey serum solution with $0.1 \%$ Triton X-100 in $1 \times$ PBS to block nonspecific binding. Tissue sections were then incubated overnight with primary antibodies at $4{ }^{\circ} \mathrm{C}$, followed by incubation with fluorescent conjugated secondary antibodies. Primary antibodies were directed against SARS-CoV-2 nucleoprotein (1:500 or 1:1,000), ACE2 (1:200), human cytokeratin 19 (1:250), CD34 (1:100), vimentin (1:100), acetylated $\alpha$-tubulin IV, CC10 (1:500), HT156 (1:150) and pro-SP-C (1:500). Secondary antibodies were: donkey anti-mouse IgG heavy and light chains-AlexaFluor 488 (1:1,000), donkey anti-rabbit IgG heavy and light chains-AlexaFluor 488(1:1,000), donkey anti-goat IgG heavy and light chains-AlexaFluor $594(1: 1,000)$, donkey anti-mouse IgG heavy and light chains-AlexaFluor Plus 594 (1:1,000), donkey anti-rabbit IgG heavy and light chains-AlexaFluor Plus 594 (1:1,000), and donkey anti-Mouse IgG heavy and light chainsAlexaFluor 647 (1:1,000). Background autofluorescence was then quenched using a $0.1 \%$ Sudan Black B solution in $80 \%$ ethanol before staining with DAPI. Slides were mounted and then imaged using an Olympus BX61 upright wide-field microscope using Volocity software (version 6.3) with a Hamamatsu ORCA RC camera. Appropriate negative controls without primary antibodies were also imaged using the same exposure time as matching stained sections. Whole-image contrast, brightness and pseudocolouring were adjusted using Imagej/Fiji (v.2.0.0-rc-69/1.51w) and Adobe Photoshop (version CS6).

\section{RNA in situ hybridization analysis of SARS-CoV-2 infection}

RNA in situ hybridization was performed on $10 \%$ formalin-fixed, paraffin-embedded 5 - $\mu \mathrm{m}$ sections of human lung tissues from LoM using the RNAscope 2.5 HD Reagent Kit according to the manufacturer's instructions (Advanced Cell Diagnostics). In brief, tissue sections 
were mounted on Superfrost Plus microscope slides (Fisher Scientific), heated at $60^{\circ} \mathrm{C}$ for $1 \mathrm{~h}$, deparaffinized in xylene $(2 \times 5 \mathrm{~min})$ and $100 \%$ ethanol $(2 \times 2 \mathrm{~min})$ and air-dried. Tissue sections were then incubated with hydrogen peroxide to block endogenous peroxidases for $10 \mathrm{~min}$ at room temperature, followed by epitope retrieval (Advanced Cell Diagnostics) for $30 \mathrm{~min}$ in a water bath at $95^{\circ} \mathrm{C}$. Subsequently, tissue sections were immediately washed in double-distilled water then dehydrated in $100 \%$ ethanol for 2 min before air-drying. Tissue sections were then incubated with Protease Plus (Advanced Cell Diagnostics) for 30 min at $40^{\circ} \mathrm{C}$ in a HybEZ hybridization oven (Advanced Cell Diagnostics). Sections were rinsed 3 times in double-distilled water, and then incubated with a prewarmed target probe designed to hybridize with the spike protein of SARS-CoV-2 (cat. no. 848561, Advanced Cell Diagnostics) for $2 \mathrm{~h}$ at $40^{\circ} \mathrm{C}$. Tissue sections were then washed and the signal amplified according to the manufacturer's instructions, and developed using alkaline phosphatase and Fast Red substrate. Tissue sections were counterstained with DAPI, mounted with Prolong Gold (Invitrogen) and imaged on an EVOS M5000 microscope (Invitrogen).

\section{Electron microscopy analysis of SARS-CoV-2 infection}

Small pieces of human lung tissue collected from SARS-CoV-2-infected LoM at 2 days after infection were fixed in $4 \%$ paraformaldehyde and $2.5 \%$ glutaraldehyde in $0.15 \mathrm{M}$ sodium phosphate buffer, $\mathrm{pH} 7.4$, for $2 \mathrm{~h}$ at room temperature. The tissues were subsequently transferred to $10 \%$ formalin for 7 days. Specimens were washed in $0.1 \mathrm{M}$ sodium cacodylate, $\mathrm{pH} 7.4$, and then post-fixed with $1 \%$ cacodylate-buffered osmium tetroxide for $1 \mathrm{~h}$. After washing in $0.05 \mathrm{M}$ sodium cacodylate buffer, pH 7.0, the samples were treated with $1 \%$ tannic acid in $0.05 \mathrm{M}$ sodium cacodylate buffer for $30 \mathrm{~min}$ to enhance tissue contrast and preserve structure ${ }^{46}$. Tissue pieces were washed in deionized water, dehydrated in ethanol and placed through two exchanges of propylene oxide before infiltration and embedment in PolyBed 812 epoxy resin (Polysciences). Semi-thin $(1 \mu \mathrm{m})$ sections of tissue blocks were cut and stained with $1 \%$ toluidine blue in $1 \%$ sodium borate for examination by light microscopy. Ultra-thin $(70 \mathrm{~nm})$ sections were cut of selected regions of interest, mounted on 200 mesh copper grids and stained with $4 \%$ aqueous uranyl acetate and Reynolds' lead citrate. Grids were observed on a JEOLJEM 1230 transmission electron microscope operating at $80 \mathrm{kV}$ (JEOL USA) and images were acquired with a Gatan Orius SC1000 CCD Digital Camera and Gatan Microscopy Suite software (v.3.0, Gatan). Virus particle sizes were measured in Fiji/Image J (v.2.0.0-rc-69/1.52p).

\section{Processing of human lung tissues from LoM for RNA-sequencing analysis}

Human lung tissues from LoM were collected in RNAlater and kept at $4{ }^{\circ} \mathrm{C}$ for $24 \mathrm{~h}$ before storage at $-80^{\circ} \mathrm{C}$ until further processing. To isolate RNA, samples stored in RNAlater were thawed and the tissue transferred to a new tube containing $1-\mathrm{mm}$ glass beads and $1 \mathrm{ml}$ Trizol. Tissues were subsequently homogenized using a MagNA Lyser (Roche) for $30 \mathrm{~s}$ at $6,000 \mathrm{rpm}$. Between rounds of homogenization, tissues were incubated on ice for $1 \mathrm{~min}$. Following tissue homogenization, Trizol homogenate was transferred to a new tube and stored at $-80^{\circ} \mathrm{C}$.

\section{RNA-sequencing analysis}

RNA was extracted from human lung samples from LoM using a Trizol Plus RNA extraction kit (Thermo Fisher), quantified using a Qubit RNA assay kit and checked for quality using a Bioanalyzer RNA600 Nano kit (Agilent). RNA integrity scores were typically 7.0 and greater. One $\mu \mathrm{g}$ of RNA was used to construct libraries for sequencing using a NEBNext Ultra II library prep kit with polyA RNA selection. Barcoded libraries were sequenced on a Novaseq $60002 \times 100 \mathrm{bp}$ following manufacturer's instructions (Illumina). Sequence quality was assessed using FASTQC (v.0.11.9). No issues were detected with the data and the quality was typical for RNA extracted from fresh-frozen material. A small amount of index hopping was detected (0.09\%), due to the single indices used in the library preparation. Raw reads were mapped to the human, mouse and SARS-CoV-2 reference genomes simultaneously (GRCh38.p13, GRCm38.p6 M25 and NC_045512, respectively) using the BBsplit function in BBmap (v.38.86). This step minimized cross-mapping of reads among genomes. There was a nonsignificant, negative relationship between the amount of viral and human RNA reads $\left(R^{2}=0.136\right)$. We then mapped and quantified on a transcript and gene model basis using STAR (v.2.7.5a) and Salmon (v.1.2.1) ${ }^{47,48}$. Reads mapping to multiple locations were dropped from analysis. On average, in the human lung tissue samples from LoM, $80 \pm 6 \%$ ( \pm s.d.) of the reads mapped to human, $19 \pm 6 \%$ ( \pm s.d.) mapped to mouse and $1 \pm 1 \%$ ( \pm s.d.) mapped to the SARS-CoV-2 genome. The per cent of virus ranged from $0.05 \%$ to $3.4 \%$ among infected mice: day-2 mice had the most virus, which is consistent with the infection titres we observed. Samples from naive mice were $95 \%$ or more human data.

\section{Statistics and reproducibility}

No statistical methods were used to predetermine sample size. No randomization was used to determine allocation of samples or mice to experimental groups and downstream analysis. The investigators were not blinded to group allocation for data collection and analysis. In Fig. 1b, titres for SARS-CoV and MERS-CoV represent two independent experiments, and titres for SARS-CoV-2, WIV1-CoV and SHC014-CoV represent one experiment. Virus titres between SARS-CoV-2-infected LoM analysed 2 days after exposure and days 6 and 14 after exposure (Fig. 1c) were compared with a two-sided Kruskal-Wallis with Dunn's multiple comparisons test; data represent one experiment. Pieces of human lung implants obtained from LoM in Fig. 1c were used for the RNAscope and immunofluorescence analysis shown in Fig. 1d-g, the histological and electron microscopy analyses in Fig. 2, and the RNA-sequencing gene-expression analyses in Fig. 3, Extended Data Fig. 3, Extended Data Tables 3-5, Supplementary Tables 1,2. The number of independent human lung tissue samples from LoM that were analysed for each parameter is indicated in the figure and table legends. RNA-sequencing data were normalized and investigated for changes in gene expression using DESeq2 package (v.3.1.1) in R (v. 3.6.3) ${ }^{49}$; statistical tests were two-sided. We focused the analysis on the naive controls versus LoM at days 2, 6 and 14 after infection. Wald's tests were performed to contrast each day with naive controls. Because mice were euthanized at each time point, we treated each day independently rather than as a time series. $P$ values were adjusted for multiple testing using a false discovery rate using the Benjamini-Hochberg method ${ }^{50}$. Data were analysed both jointly and within each treatment compared to naive controls. Differential expression of outliers was assessed and found to be insignificant in overall effect. Graphs and summary tables were built in R using ggplot; gene-set enrichment was performed using gene-set enrichment analysis and Gene Ontology (GO) analysis (tidyverse 1.3.0, PCATools 1.2.0, Sqldf 0.4-11, na.tools 0.3.1, ggbiplot 0.55, ggplot2 3.3.1 and dplyr 0.8.4). The statistical significance of enrichment scores calculated by gene-set enrichment analysis was determined using an empirical phenotype-based permutation test ${ }^{51}$, and $P$ values were adjusted for multiple testing using a false discovery rate using the Benjamini-Hochberg method ${ }^{50}$. Specific gene sets of interest were then investigated for patterns of expression across treatment and time using unsupervised clustering of normalized gene-expression counts. GO analysis and visualization were performed with Gorilla (no version) $^{52}$. In Fig. 4, virus titres in LoM dosed with vehicle control or EIDD-2801 were compared with a two-sided Mann-Whitney $U$ test (GraphPad Prism v.8.0 or R v.3.5.3). The effect of EIDD-2801 treatment on virus titres (Fig. 4a, b) represents one experiment and the effect of EIDD-2801 prophylaxis (Fig. 4c-e) represents two independent experiments. All independent attempts of repetition were completed with similar results. 


\section{Reporting summary}

Further information on research design is available in the Nature Research Reporting Summary linked to this paper.

\section{Data availability}

Gene-expression data are available at the Gene Expression Omnibus repository (accession number GSE155286). All other data are available from the corresponding author on reasonable request. Publicly available datasets used in this study were: (1) the sequence of the SARS-CoV-2 strain 2019-nCoV/USA-WA1/2020 (GenBank accession number MN985325.1) and (2) human lung tissue gene-expression data profiled by the GTEx project (https://gtexportal.org/home/; data retrieved 20 October 2020 and 3 November 2020). Source data are provided with this paper.

44. Scobey, T. et al. Reverse genetics with a full-length infectious cDNA of the Middle East respiratory syndrome coronavirus. Proc. Natl Acad. Sci. USA 110, 16157-16162 (2013).

45. Yount, B. et al. Reverse genetics with a full-length infectious cDNA of severe acute respiratory syndrome coronavirus. Proc. Natl Acad. Sci. USA 100, 12995-13000 (2003).

46. Simionescu, N. \& Simionescu, M. Galloylglucoses of low molecular weight as mordant in electron microscopy. I. Procedure, and evidence for mordanting effect. J. Cell Biol. 70, 608-621 (1976).

47. Dobin, A. et al. STAR: ultrafast universal RNA-seq aligner. Bioinformatics 29, 15-21 (2013).

48. Patro, R., Duggal, G., Love, M. I., Irizarry, R. A. \& Kingsford, C. Salmon provides fast and bias-aware quantification of transcript expression. Nat. Methods 14, 417-419 (2017).

49. Love, M. I., Huber, W. \& Anders, S. Moderated estimation of fold change and dispersion for RNA-seq data with DESeq2. Genome Biol. 15, 550 (2014).

50. Benjamini, Y. \& Hochberg, Y. Controlling the false discovery rate: a practical and powerful approach to multiple testing. J. R. Stat. Soc. 57, 289-300, (1995).

51. Subramanian, A. et al. Gene set enrichment analysis: a knowledge-based approach for interpreting genome-wide expression profiles. Proc. Natl Acad. Sci. USA 102, 15545-15550 (2005).

52. Eden, E., Navon, R., Steinfeld, I., Lipson, D. \& Yakhini, Z. GOrilla: a tool for discovery and visualization of enriched $\mathrm{GO}$ terms in ranked gene lists. BMC Bioinformatics 10, 48 (2009).
Acknowledgements We thank current and past members of the laboratory of J.V.G. for technical assistance; technicians at the UNC Animal Histopathology and Laboratory Medicine Core and Division of Comparative Medicine; the UNC School of Medicine Bioinformatics and Analytics Research Collaborative (BARC) for providing technical support; and K. Mollan and B. Shook-Sa of the UNC CFAR Biostatistics Core for providing statistical support. This work was supported by funding from National Institutes of Health grants R21Al113736 (R.J.P.), U19Al100625 and R01 Al108197 (R.S.B.), Fast Grants (L.E.G.), RO1Al123010 (A.W.), RO1Al111899 (J.V.G.), R01Al140799 (J.V.G.) and R01MH108179 (J.V.G.). This project was also supported by the North Carolina Policy Collaboratory at the University of North Carolina at Chapel Hill with funding from the North Carolina Coronavirus Relief Fund established and appropriated by the North Carolina General Assembly. The Microscopy Services Laboratory, Department of Pathology and Laboratory Medicine, is supported in part by P30 CA016086 Cancer Center Support Grant to the UNC Lineberger Comprehensive Cancer Center.

Author contributions A.W., C.E.J., W.Y., M.K. and C.D. constructed LoM. A.W. contributed to experimental design, data interpretation, data presentation and manuscript writing, and coordinated the study and the preparation of the manuscript. L.E.G. performed the virus inoculations, animal necropsies, virus titring and processing of lung tissues for RNA extraction, and contributed to the experimental design, planning, data analysis, data interpretation and manuscript writing. C.E.J. performed immunofluorescence and H\&E analyses, W.Y. performed immunohistochemistry and $\mathrm{H} \& \mathrm{E}$ analyses, and M.K. performed the in situ hybridization analysis of LoM human lung tissues. K.H.D., A.S., S.R.L. and K.G. assisted with the in vivo experiments with coronavirus-infected LoM. V.J.M. in conjunction with K.K.W. performed the electron microscopy analysis. G.R.B., A.A.K., M.G.N. and G.P. assisted with the EIDD-2801 experiments. F.B.A. assisted with the pathohistological analysis. H.L., H.M.K., T.Z. and P.O.G. performed the RNA-sequencing analysis. E.P.B. and C.D.J. contributed to the design of RNA-sequencing experiments. R.J.P. assisted with the immunofluorescence analysis, and contributed to experimental design, data interpretation, data presentation and manuscript writing. R.S.B. conceived and designed experiments, and contributed to data interpretation and manuscript writing. J.V.G. conceived, designed and coordinated the study, conceived and designed experiments, and contributed to data interpretation, data presentation and manuscript preparation.

Competing interests G.P., M.G.N., G.R.B. and A.A.K. are employees of Emory and have a financial interest in molnupiravir (EIDD-2801).

\section{Additional information}

Supplementary information The online version contains supplementary material available at https://doi.org/10.1038/s41586-021-03312-w.

Correspondence and requests for materials should be addressed to J.V.G.

Peer review information Nature thanks the anonymous reviewers for their contribution to the peer review of this work.

Reprints and permissions information is available at http://www.nature.com/reprints. 


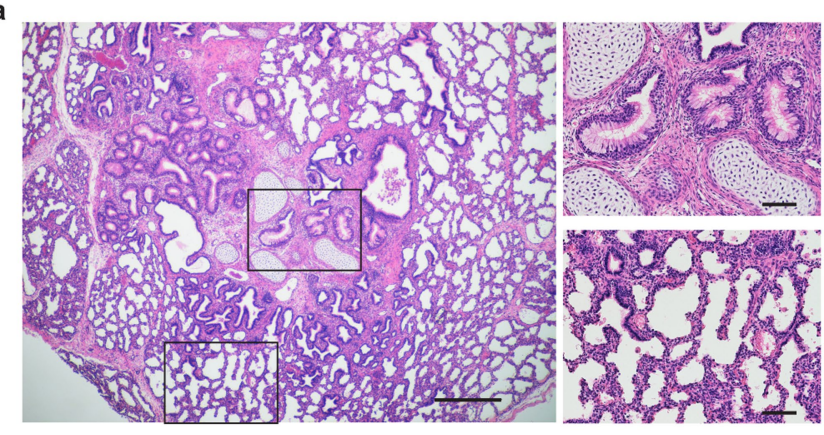

b

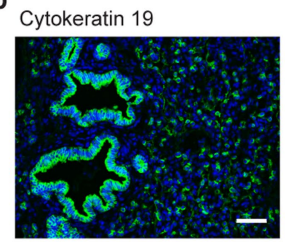

d

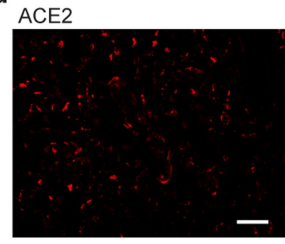

CD34

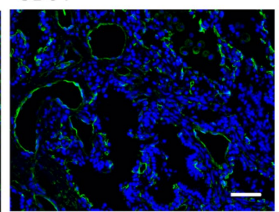

Cytokeratin 19

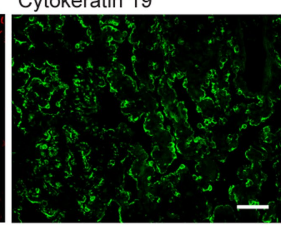

Extended Data Fig. 1 | Human epithelial cells in the human lung tissue of

LoM express ACE2. a, H\&E staining of the human lung tissue of a naive

$\operatorname{LoM}(n=6)$. Boxes in the left panel indicate regions that are shown in

higher-magnification images in the right panels, of cartilaginous airways (top)

and noncartilaginous airways and alveoli (bottom). Scale bars, $500 \mu \mathrm{m}$ (left),

$100 \mu \mathrm{m}$ (top right, bottom right). b. Immunofluorescence staining for human

cytokeratin 19 (epithelial cells, green; nuclei, blue), CD34 (endothelial cells,

green; nuclei, blue) or vimentin (mesenchymal cells, green; nuclei, blue) $(n=8)$.
Vimentin

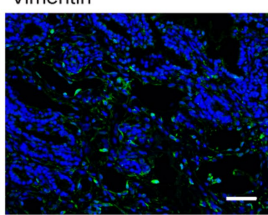

ACE2

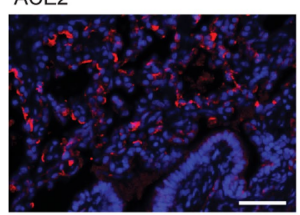

e

Merged

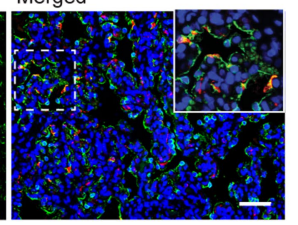

Scale bars, $50 \mu \mathrm{m}$. c, Immunofluorescence staining for human ACE2 in the human lung tissue of a naive LoM (positive cells, red; nuclei, blue) $(n=9)$. Scale bar, $50 \mu \mathrm{m}$. d, Costaining for human ACE2 (positive cells, red) and cytokeratin 19 (positive cells, green) in human lung tissue from naive LoM (nuclei, blue) $(n=9)$. Scale bars, $50 \mu \mathrm{m}$. e, Immunohistochemical staining for TMPRSS2 in human lung tissue from naive LoM (positive cells, brown) $(n=6)$. Scale bar, $100 \mu \mathrm{m} . n$, number of biologically independent lung tissues analysed. 


\section{Article}

a

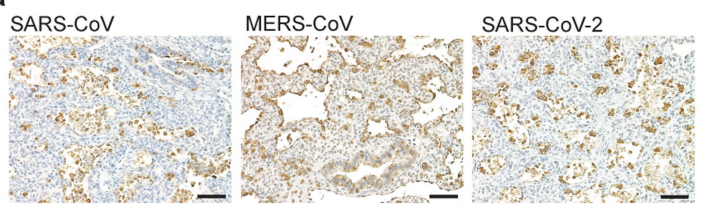

b

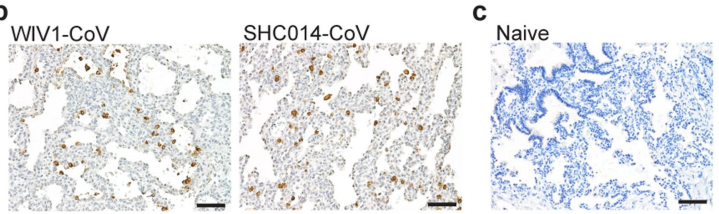

Extended Data Fig. 2 | Viral nucleoprotein in the human lung tissues of LoM infected with recently emerged human coronaviruses and bat

coronaviruses. a-c, Immunohistochemical staining for virus nucleoprotein in human lung tissue collected from LoM two days after exposure to the recently emerged human coronaviruses SARS- $\operatorname{CoV}(n=4), \operatorname{MERS}-\operatorname{CoV}(n=5)$ or SARS-CoV-2 $(n=6)(a)$, LoM two days after exposure to the bat coronaviruses WIV1-CoV or SHC014-CoV $(n=10)$ (b) or naive LoM $(n=6)$ (c). Positive cells, brown. Scale bars, $100 \mu \mathrm{m}$. $n$, number of biologically independent lung tissues analysed. 
a

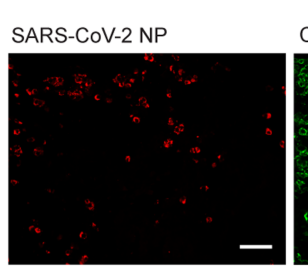

SARS-CoV-2 NP

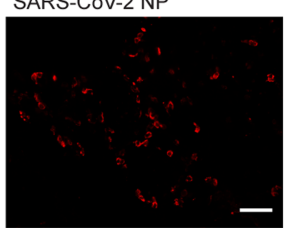

b

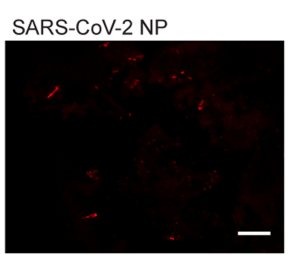

SARS-CoV-2 NP
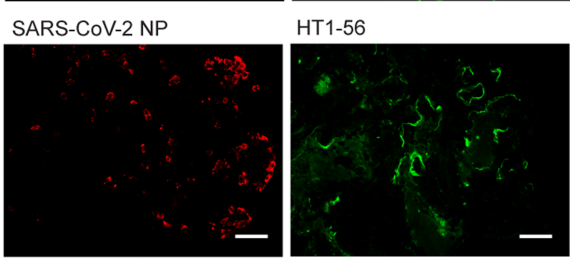

$\alpha$-Tubulin

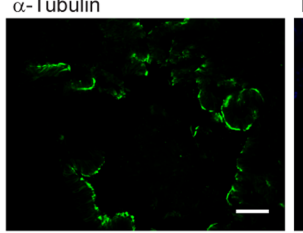

HT1-56

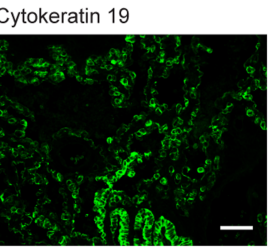

CD34

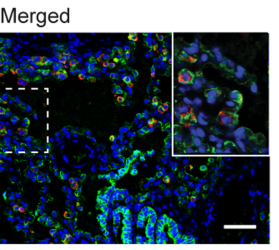

Merged
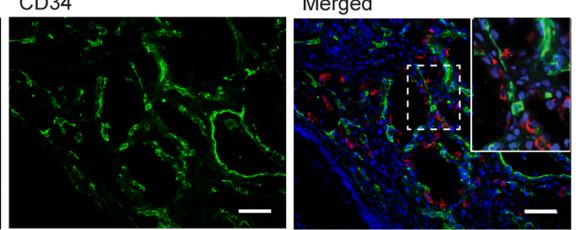

Merged

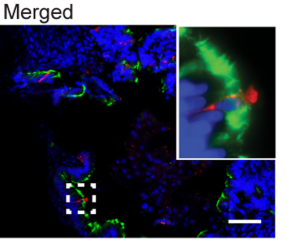

Merged

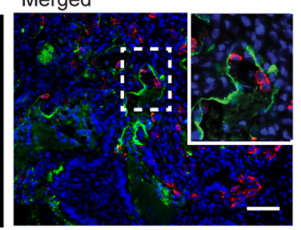

Extended Data Fig. 3 |SARS-CoV-2 infection of human epithelial cell types in the human lung tissue of LoM. a, b, Single-colour and merged images of those shown in Fig. 1f, g, depicting costaining of human lung tissue from LoM two days after exposure to SARS-CoV-2 for virus nucleoprotein (red) and cytokeratin 19 (epithelial cells, green) $(n=6)$, CD34 (endothelial cells, green)
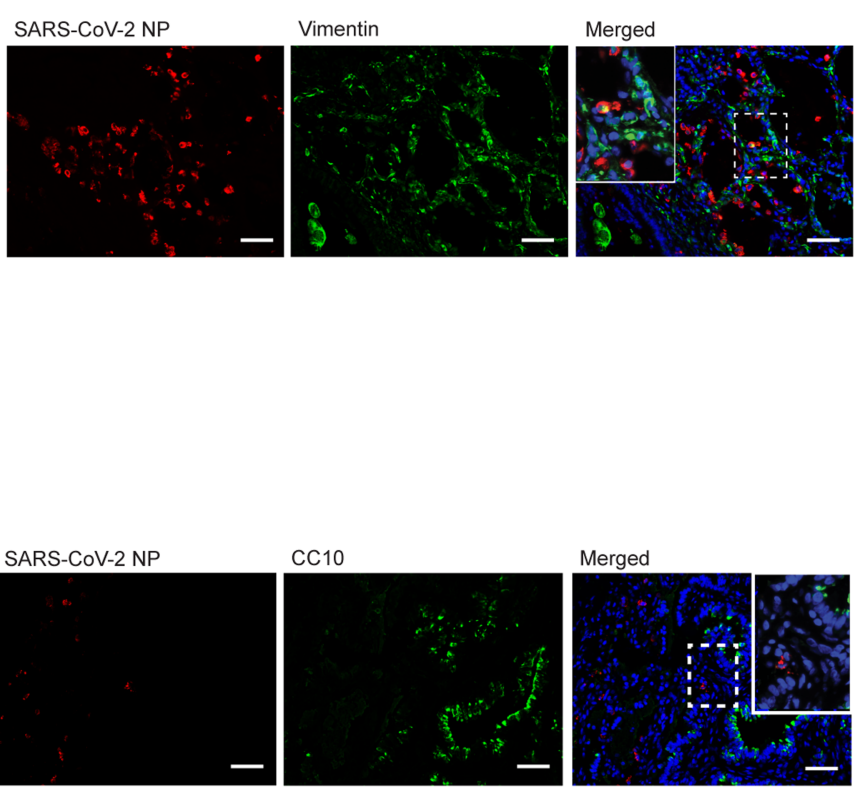

SARS-COV-2 NP Pro-SP-C Merged
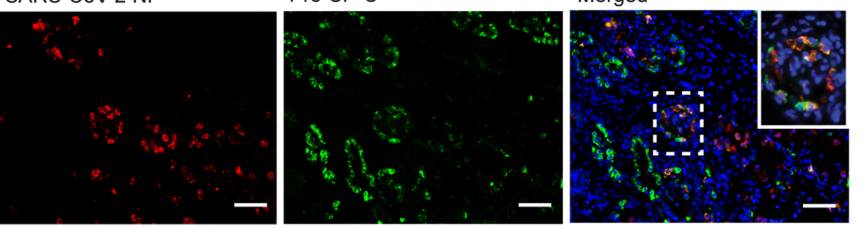

$(n=4)$ or vimentin (mesenchymal cells, green) $(n=4)(a)$ or acetylated $\alpha$-tubulin IV (ciliated cells, green) $(n=6), \mathrm{CC10}$ (club cells, green) $(n=6), \mathrm{HT1}-56$ (alveolar type-1 pneumocytes, green) $(n=6)$ or pro-SP-C (alveolar type-2 pneumocytes, green) $(n=3)$ (b). Nuclei, blue. Scale bars, $50 \mu \mathrm{m} . n$, number of biologically independent lung tissues analysed. 


\section{Article}
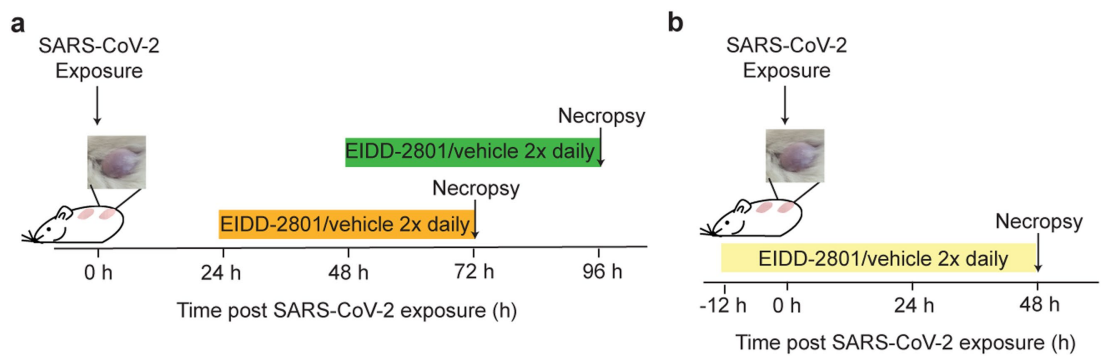

Extended Data Fig. 4 | Experimental design for the evaluation of EIDD-2801 as a treatment or pre-exposure prophylaxis for infection with SARS-CoV-2. a, Experimental design for treatment. LoM were orally administered EIDD-2801 or vehicle at $24 \mathrm{~h}$ or $48 \mathrm{~h}$ after exposure to SARS-CoV-2, and every $12 \mathrm{~h}$ thereafter. Virus titres were measured two days after the initiation of

treatment.b, Experimental design for pre-exposure prophylaxis. LoM were orally administered EIDD-2801 or vehicle control $12 \mathrm{~h}$ before exposure to SARS-CoV-2, and every $12 \mathrm{~h}$ thereafter. Virus titres in human lung tissues were measured two days after exposure. 
Extended Data Table 1 | Description of Lo used for the analysis of human and bat coronavirus replication

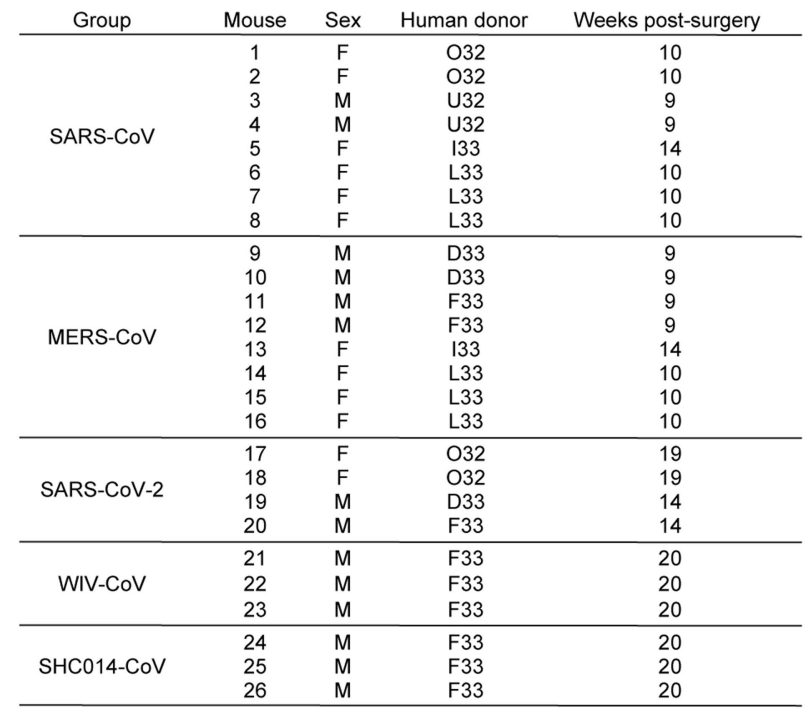




\section{Article}

Extended Data Table 2 | Description of LoM used for the analysis of SARS-CoV-2 replication and pathogenesis over time

\begin{tabular}{ccccc} 
Group & Mouse & Sex & Human donor & Weeks post-surgery \\
\hline \multirow{5}{*}{ Day 2 } & 27 & $\mathrm{~F}$ & $\mathrm{~A} 31$ & 59 \\
& 28 & $\mathrm{~F}$ & $\mathrm{~A} 31$ & 59 \\
& 29 & $\mathrm{~F}$ & $\mathrm{~F} 32$ & 25 \\
& 30 & $\mathrm{~F}$ & $\mathrm{I} 32$ & 19 \\
\hline \multirow{5}{*}{ Day 6 } & 31 & $\mathrm{~F}$ & $\mathrm{R} 30$ & 74 \\
& 32 & $\mathrm{~F}$ & $\mathrm{~A} 31$ & 59 \\
& 33 & $\mathrm{~F}$ & $\mathrm{I} 32$ & 19 \\
& 34 & $\mathrm{~F}$ & $\mathrm{~F} 32$ & 25 \\
& 35 & $\mathrm{~F}$ & $\mathrm{~F} 32$ & 25 \\
\hline \multirow{5}{*}{ Day 14 } & 36 & $\mathrm{~F}$ & $\mathrm{~A} 31$ & 59 \\
& 37 & $\mathrm{~F}$ & $\mathrm{I} 32$ & 19 \\
& 38 & $\mathrm{~F}$ & $\mathrm{R} 30$ & 74 \\
& 39 & $\mathrm{~F}$ & $\mathrm{~F} 32$ & 25 \\
& 40 & $\mathrm{~F}$ & $\mathrm{~F} 32$ & 25
\end{tabular}


Extended Data Table 3 | Abundance of viral transcripts detected in Lo infected with SARS-CoV-2

\begin{tabular}{cccccc} 
Group & Mouse & Sex & Human donor & Weeks post-surgery & \%Viral transcripts \\
\hline \multirow{2}{*}{ Day 2 } & 1 & $\mathrm{~F}$ & $\mathrm{~A} 31$ & 59 & $0.55301 \%$ \\
& 41 & $\mathrm{~F}$ & I32 & 19 & $3.55979 \%$ \\
\hline \multirow{3}{*}{ Day 6 } & 31 & $\mathrm{~F}$ & $\mathrm{R} 30$ & 74 & $1.01818 \%$ \\
& 32 & $\mathrm{~F}$ & $\mathrm{~A} 31$ & 59 & $0.46368 \%$ \\
& 33 & $\mathrm{~F}$ & $\mathrm{I} 2$ & 19 & $1.38159 \%$ \\
\hline \multirow{3}{*}{ Day 14 } & 37 & $\mathrm{~F}$ & $\mathrm{I} 2$ & 19 & $0.69389 \%$ \\
& 39 & $\mathrm{~F}$ & $\mathrm{~F} 32$ & 25 & $0.05856 \%$ \\
& 42 & $\mathrm{~F}$ & $\mathrm{~A} 31$ & 59 & $0.18557 \%$ \\
\hline \multirow{4}{*}{ Naive } & 43 & $\mathrm{~F}$ & $\mathrm{~A} 31$ & 59 & $\mathrm{~N} / \mathrm{A}$ \\
& 44 & $\mathrm{~F}$ & I32 & 19 & $\mathrm{~N} / \mathrm{A}$ \\
& 45 & $\mathrm{~F}$ & I32 & 19 & $\mathrm{~N} / \mathrm{A}$ \\
& 46 & $\mathrm{~F}$ & $\mathrm{I32}$ & 19 & \\
\hline
\end{tabular}

The per cent viral transcripts of total transcripts sequenced from SARS-CoV-2-infected $(n=8)$ and naive $(n=4)$ Lo used for RNA-sequencing analysis is shown. Using a linear model, there was no effect of human donor on viral transcript abundance $(\operatorname{lm}()$ in R: estimate -0.012 , $P=0.103) . n$, number of biologically independent lung tissues analysed. N/A, not applicable. 


\section{Article}

Extended Data Table 4 | Abundance of human ACE2 and

TMPRSS2 transcripts detected in LoM infected with

SARS-CoV-2

\begin{tabular}{cccccc} 
Group & Mouse & Humandonor & GAPDH (TPM) & ACE2 (TPM) & TMPRSS2 (TPM) \\
\hline \multirow{2}{*}{ Day 2 } & 1 & A31 & 517.41 & 1.79 & 33.54 \\
& 41 & I32 & 432.19 & 4.63 & 41.96 \\
\hline \multirow{2}{*}{ Day 6 } & 31 & R30 & 654.63 & 2.67 & 78.64 \\
& 32 & A31 & 784.39 & 0.73 & 21.62 \\
& 33 & I32 & 481.94 & 1.56 & 41.07 \\
\hline \multirow{2}{*}{ Day 14 } & 37 & I32 & 419.55 & 2.02 & 38.94 \\
& 39 & F32 & 492.57 & 1.90 & 61.95 \\
& 42 & A31 & 351.54 & 1.54 & 43.50 \\
\hline \multirow{2}{*}{ Naive } & 43 & A31 & 2384.62 & 0.19 & 15.35 \\
& 44 & I32 & 363.42 & 0.82 & 38.95 \\
& 45 & I32 & 359.21 & 0.67 & 38.19 \\
& 46 & I32 & 339.77 & 0.89 & 32.93 \\
\hline
\end{tabular}

The abundance of human GAPDH, ACE2 and TMPRSS2 transcripts sequenced in human lung tissue collected from naive $(n=4)$ and SARS-CoV-2-infected $(n=8)$ LoM is shown. The median ACE2 and TMPRSS2 expression in human lung tissue of naive mice was 0.75 and 35.6 TPM, respectively, which is comparable to the median expression of ACE2 (1.01 TPM) and TMPRSS2 (43.2 TPM) observed in human lung tissue profiled by the GTEx project (https://gtexportal.org/ home/; data retrieved 20 October 2020 and 3 November 2020). No effect of human donor on ACE2 expression ( $F=2.06259641 ; P=0.1756$ via two-sided analysis of variance) was observed. Approximately the same amount of variation was observed within a human donor (mean variance within donors $=4.82$ ) as across all donors (variance $=4.47$ ). $n$, number of biologically independent lung tissues analysed. 
Extended Data Table 5 | Human interferon and cytokine genes upregulated during infection with SARS-CoV-2

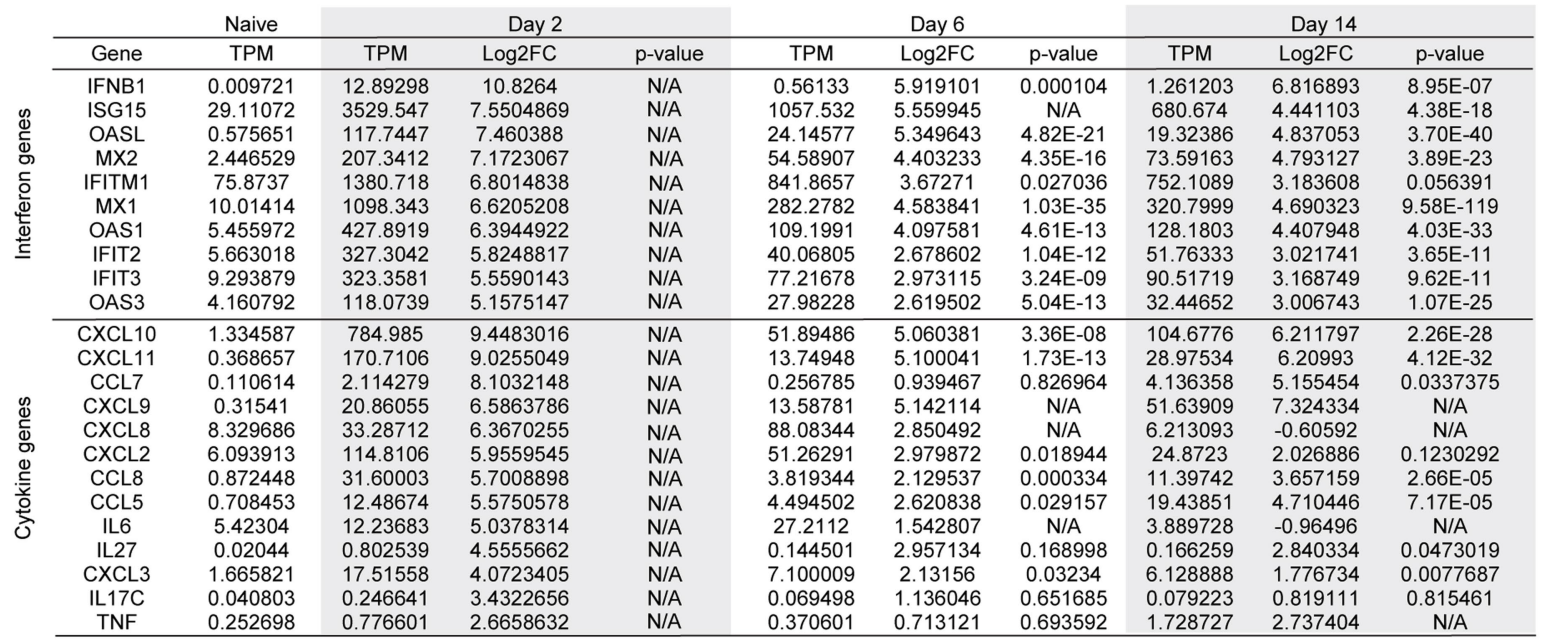

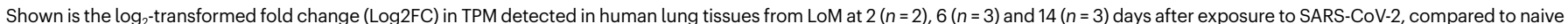
control mice $(n=4)$. $P$ values were calculated in DESeq2 using a two-sided Wald's test and adjusted for multiple testing using a false discovery rate. For samples marked not applicable (N/A), DESeq2 could not calculate a $P$ value owing to sample size or the variance between samples. $n$, number of biologically independent lung tissues analysed. 
Article

Extended Data Table 6 | Description of Lo used to evaluate the efficacy of EIDD-2801 in the treatment of SARS-CoV-2 infection or as pre-exposure prophylaxis

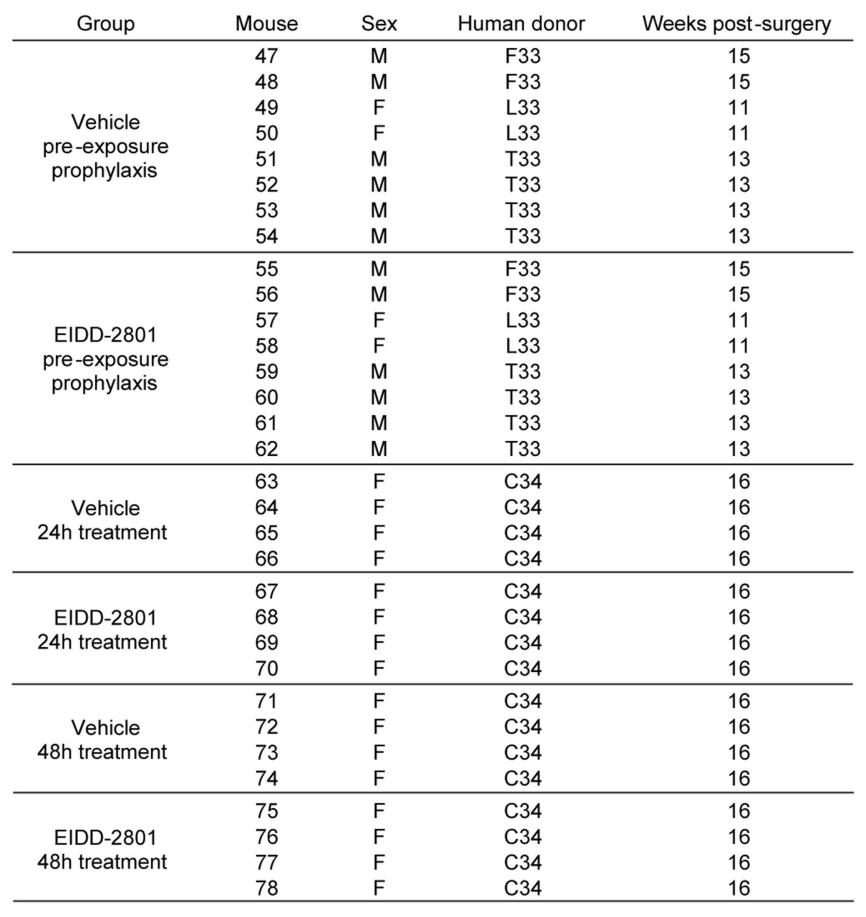




\section{Reporting Summary}

Nature Research wishes to improve the reproducibility of the work that we publish. This form provides structure for consistency and transparency in reporting. For further information on Nature Research policies, see our Editorial Policies and the Editorial Policy Checklist.

\section{Statistics}

For all statistical analyses, confirm that the following items are present in the figure legend, table legend, main text, or Methods section.

n/a Confirmed

$\square$ The exact sample size $(n)$ for each experimental group/condition, given as a discrete number and unit of measurement

$\square$ A statement on whether measurements were taken from distinct samples or whether the same sample was measured repeatedly

The statistical test(s) used AND whether they are one- or two-sided

$\square$ Only common tests should be described solely by name; describe more complex techniques in the Methods section.

Х $\square$ A description of all covariates tested

$\square$ \A description of any assumptions or corrections, such as tests of normality and adjustment for multiple comparisons

$\square$ A full description of the statistical parameters including central tendency (e.g. means) or other basic estimates (e.g. regression coefficient)

$\bigotimes$ AND variation (e.g. standard deviation) or associated estimates of uncertainty (e.g. confidence intervals)

$\varnothing$ For null hypothesis testing, the test statistic (e.g. $F, t, r$ ) with confidence intervals, effect sizes, degrees of freedom and $P$ value noted

Give $P$ values as exact values whenever suitable.

Х $\square$ For Bayesian analysis, information on the choice of priors and Markov chain Monte Carlo settings

Х $\square$ For hierarchical and complex designs, identification of the appropriate level for tests and full reporting of outcomes

Х $\square$ Estimates of effect sizes (e.g. Cohen's $d$, Pearson's $r$ ), indicating how they were calculated

Our web collection on statistics for biologists contains articles on many of the points above.

\section{Software and code}

Policy information about availability of computer code

Data collection Nikon Elements BR software (version 4.30.01), Volocity software (version 6.3), Gatan Microscopy Suite software (version 3.0)

Data analysis ImageJ/Fiji (version 2.0-rc-69/1.51w), Adobe Photoshop (version C S6), FASTQC (version 0.11.9), BBmap (version 38.86), STAR (version 2.7.5a), Salmon (version 1.2.1), DESeq2 (version 3.1.1), R (version 3.5.3 or 3.6.3), GOrilla (no version), tidyverse (version 1.3.0), PCATools (version

1.2.0), Sqldf (version 0.4-11), na.tools (version 0.3.1), ggbiplot (version 0.55), ggplot2 (version 3.3.1), dplyr (version 0.8.4), Graphpad Prism (version 8.0.0)

For manuscripts utilizing custom algorithms or software that are central to the research but not yet described in published literature, software must be made available to editors and reviewers. We strongly encourage code deposition in a community repository (e.g. GitHub). See the Nature Research guidelines for submitting code \& software for further information.

\section{Data}

Policy information about availability of data

All manuscripts must include a data availability statement. This statement should provide the following information, where applicable:

- Accession codes, unique identifiers, or web links for publicly available datasets

- A list of figures that have associated raw data

- A description of any restrictions on data availability

Gene-expression data are available at the Gene Expression Omnibus (GEO) repository (accession: GSE155286). Source data is provided for Fig. 1b,c and Fig. 4a-d. All other data is available from corresponding authors on reasonable request. Publicly available data sets used in this study: 1) sequence of the SARS-CoV-2 strain $2019-$ nCoV/USA-WA1/2020 (GenBank accession: MN985325.1) and 2) human lung tissue gene expression data profiled by the GTEx project (https://gtexportal.org/ home/; data retrieved 10/20/2020 and 11/3/2020). 


\section{Field-specific reporting}

Please select the one below that is the best fit for your research. If you are not sure, read the appropriate sections before making your selection.

$\bigotimes$ Life sciences $\quad \square$ Behavioural \& social sciences $\quad \square$ Ecological, evolutionary \& environmental sciences

For a reference copy of the document with all sections, see nature.com/documents/nr-reporting-summary-flat.pdf

\section{Life sciences study design}

All studies must disclose on these points even when the disclosure is negative.

Sample size No statistical methods were used to predetermine sample size. To aid statistical analysis, at least 3 samples were used for each group, unless otherwise noted. At least three animals were utilized for each virus and time point. The number of animals per group was also influenced by the number different analyses performed for each experiment (e.g. virus titer, immunofluorescence/immunohistochemistry, electron microscopy, and/or RNA-sequencing) and so that at least three animals per group were represented for each downstream analysis unless otherwise noted.

Data exclusions No data was excluded from analysis.

Replication For the analysis of coronavirus replication in LoM, SARS-CoV and MERS-CoV infections represented 4 human donor cohorts. For the analysis of SARS-CoV-2 replication over time, 4 human donor cohorts were represented. For the analysis of the protective efficacy of EIDD-2801, 3 human donor cohorts were represented and the experiment performed twice. All attempts at replication were successful.

Randomization No randomization was used to determine how samples/animals were allocated to experimental groups or processed. Mice were allocated to groups based on their implant sizes and their donor cohort to keep experimental groups relatively equivalent with these covariates. Whole implants or pieces of implants (depending on size) from each animal were allocated for analysis by virus titer, immunofluorescence/ immunohistochemistry, electron microscopy, and/or RNA-sequencing so that at least three animals per group were represented (with the exception of the day 2 RNA-seq analysis) and human donor cohorts were represented as equivalently.

Blinding

The investigators were not blinded to group allocation for data collection and analysis. For virus titer measurements, the infecting strain determines what type of cells are used for plaque assays. For example, MERS-CoV is titered on Vero CCL81 cells while SARS-CoV, SARS-CoV-2, WIV-1, and SHCO14 are titered using Vero E6 cells. For the RNA-seq analysis, viral and human gene transcripts were analyzed from the same sample. For RNAscope, immunoflourescense, and immunohistochemistry the infecting strain also determines which RNA probe or antibody is used to analyze tissues sections for detection of virus infected cells. H/E staining was often done in parallel. Due to COVID-19 restrictions, personnel who performed histological assays also performed data analysis. For electron microscopy analysis, only SARS-CoV-2 infected human lung tissues were analyzed. Personnel at the UNC Animal Histopathology and Clinical Chemistry Core who performed MSB staining were not privy to group allocations.

\section{Reporting for specific materials, systems and methods}

We require information from authors about some types of materials, experimental systems and methods used in many studies. Here, indicate whether each material, system or method listed is relevant to your study. If you are not sure if a list item applies to your research, read the appropriate section before selecting a response.

\begin{tabular}{l|l} 
Materials \& experimental system \\
\hline $\mathrm{n} / \mathrm{a}$ & Involved in the study \\
$\square$ & $\bigotimes$ Antibodies \\
$\square$ & $\square$ Eukaryotic cell lines \\
$\square$ & $\square$ Clinical data \\
$\square$ & $\square$ Dual use research of concern
\end{tabular}

\begin{tabular}{|c|c|}
\hline$n / a$ & Involved in the study \\
\hline Х & ChIP-seq \\
\hline Х & Flow cytometry \\
\hline Х & MRI-based neuroimaging \\
\hline
\end{tabular}

\section{Antibodies}

Antibodies used
anti-ACE2; clone: Polyclonal IgG; dilution 1:200 dilution; supplier: R\&D systems; catalog: AF933 ; lot: HOK0320032; application: IF; https://www.rndsystems.com/products/human-hamster-ace-2-antibody_af933

anti-Acetylated alpha-tubulin; clone: 6-11B-1; dilution: 1:500; supplier: Zymed; catalog: 32-2700; lot: 60806682R; application: IF; https://www.thermofisher.com/antibody/product/Acetyl-alpha-Tubulin-Lys40-Antibody-clone-6-11B-1-Monoclonal/32-2700

anti-CC10; clone: E-11; dilution: 1:500; supplier: Santa Cruz Biotech; catalog: SC-365992; lots: B2219, K10106; application: IF; https:// www.scbt.com/p/cc10-antibody-e-11? requestFrom=search 
anti-CD34; clone: QBEnd10; dilution: 1:100; supplier: Dako; catalog: M7165; lot: 20011397; application: IF; https://www.agilent.com/ store/productDetail.jsp?catalogld=M716501-2

anti-Cytokeratin19; clone: A53-B/A2; dilution: 1:250; supplier: Abcam; catalog: ab7754; lot: GR3226692-4; application: IF; https:// www.abcam.com/cytokeratin-19-antibody-a53-ba2-cytoskeleton-marker-ab7754.html

anti-HT1-56; dilution: 1:150; supplier: Terrace Biotech; catalog: TB-29; lot: AHT1-56 HM29; application: IF; https:// www.terracebiotech.com/antibodies

anti-MERS nucleocapsid; clone: Polyclonal; dilution: 1:2,000; supplier: LS Bio; catalog: LS-C483529; lot: 127989; application: IHC; https://www.Isbio.com/antibodies/mers-cov-nucleoprotein-antibody-elisa-wb-western-Is-c483529/496447

anti-Pro-SP-C; clone: Polyclonal; dilution: 1:500; supplier: Seven Hills; catalog: WRAB9337; lots: 458, 0206A; application: IF; https:// www.sevenhillsbioreagents.com/products/anti-pro-sp-c-rabbit-n-terminal?_pos=1\&_sid=51b111a78\&_ss=r

anti-SARS nucleocapsid; clone: Polyclonal IgG; dilution: 1:500; supplier: Invitrogen; catalog: PA1-41098, lot: VC2962205; application: IF and IHC; https://www.thermofisher.com/antibody/product/SARS-Coronavirus-Nucleocapsid-Antibody-Polyclonal/PA1-41098

anti-SARS nucleocapsid; dilution: 1:1,000; supplier: Baric Laboratory; application: IF

anti-TMPRSS2; clone: Polyclonal Ig; dilution: 1:100; supplier: MyBioSource; catalog: MBS9215011, lot:SH200730AC; application: IHC https://www.mybiosource.com/polyclonal-human-antibody/tmprss2/9215011

anti-Vimentin; clone: V9; dilution: 1:100; supplier: Abcam; catalog: ab8069; lot: GR3226692-4; application: IF; https:// www.abcam.com/vimentin-antibody-v9-cytoskeleton-marker-ab8069.html

anti-Mouse IgG heavy and light chains-AlexaFluor 488; clone: donkey polyclonal IgG; dilution: 1:1,000; supplier: Invitrogen; catalog A21202; lot: 2147618; application: IF; https://www.thermofisher.com/antibody/product/Donkey-anti-Mouse-lgG-H-L-Highly-CrossAdsorbed-Secondary-Antibody-Polyclonal/A-21202

anti-Rabbit IgG heavy and light chains-AlexaFluor 488; clone: donkey polyclonal IgG; dilution: 1:1,000; supplier: Invitrogen; catalog: A21206; lot: 2156521; application: IF; https://www.thermofisher.com/antibody/product/Donkey-anti-Rabbit-lgG-H-L-Highly-CrossAdsorbed-Secondary-Antibody-Polyclonal/A-21206

anti-Goat IgG heavy and light chains-AlexaFluor 594; clone: donkey Polyclonal IgG; dilution: 1:1,000; supplier: Invitrogen; catalog: A11058; supplier: 2045324; application: IF; https://www.thermofisher.com/antibody/product/Donkey-anti-Goat-IgG-H-L-CrossAdsorbed-Secondary-Antibody-Polyclonal/A-11058

anti-Mouse IgG heavy and light chains-AlexaFluor Plus 594; clone: donkey Polyclonal IgG; dilution: 1:1,000; supplier: Invitrogen A32744; catalog: UL292347; application: IF; https://www.thermofisher.com/antibody/product/Donkey-anti-Mouse-IgG-H-L-HighlyCross-Adsorbed-Secondary-Antibody-Polyclonal/A32744

anti-Rabbit IgG heavy and light chains-AlexaFluor Plus 594; clone: donkey Polyclonal IgG; dilution: 1:1,000; supplier: Invitrogen A32754; catalog: VB292347; application: IF; https://www.thermofisher.com/antibody/product/Donkey-anti-Rabbit-IgG-H-L-HighlyCross-Adsorbed-Secondary-Antibody-Polyclonal/A32754

anti-Mouse IgG heavy and light chains-AlexaFluor 647; clone donkey Polyclonal IgG; dilution: 1:1,000; supplier: Invitrogen; catalog: A31571; lot: 2045337; application: IF; https://www.thermofisher.com/antibody/product/Donkey-anti-Mouse-lgG-H-L-Highly-CrossAdsorbed-Secondary-Antibody-Polyclonal/A-31571

Validation

The specificity of the antibodies purchased from commercial sources (R\&D Systems, Zymed, Santa Cruz Biotech, Dako, Abcam, Terrace Biotech, LS Bio, Seven Hills, and Invitrogen) were validated by the manufacturer as noted on their website (links provided above for each antibody). anti-SARS nucleoprotein mouse serum was produced by the Baric laboratory and validated for immunohistochemistry and immunofluorescence using COVID-19 autopsy lung sections and SARS-CoV-2 infected human airway epithelial cell cultures (https://www.cell.com/cell/pdf/s0092-8674\%2820\%2930675-9.pdf).

\section{Eukaryotic cell lines}

Policy information about cell lines

Cell line source(s)

Vero E6 cells and Vero CCL81 cells were purchased from ATCC

Authentication

Cell lines were authenticated by morphological identification and virus susceptibility profiles.

Mycoplasma contamination

Cell lines were tested negative for mycoplasma by the supplier.

Commonly misidentified lines (See ICLAC register)

No commonly misidentified cell lines were used 


\section{Animals and other organisms}

Policy information about studies involving animals; ARRIVE guidelines recommended for reporting animal research
Laboratory animals Lung-only mice (LoM) were constructed using 12-21 week old male and female NOD.Cg-Prkdcscid II2rgtm1Wj//SzJ mice (NSG; The Jackson Laboratory, Bar Harbor, ME) mice. Mice were housed in a temperature $\left(20-23^{\circ} \mathrm{C}\right)$ and humidity $(30-70 \%)$ controlled room on a 12 hour light/12 hour dark schedule.

Wild animals The study did not involve wild animals.

Field-collected samples The study did not involve field collected samples.

Ethics oversight Animal studies were carried out according to protocols approved by the Institutional Use and Care Committee at UNC-Chapel Hill and in adherence to the NIH Guide for the Care and Use of Laboratory Animals.

Note that full information on the approval of the study protocol must also be provided in the manuscript. 